\title{
Extending Conceptualisation Modes for Generalised Formal Concept Analysis
}

\author{
Francisco J. Valverde-Albacete*, Carmen Peláez-Moreno \\ Departamento de Teoría de la Señal y de las Comunicaciones, \\ Universidad Carlos III de Madrid, 28911 Leganés, Spain
}

\begin{abstract}
Formal Concept Analysis (FCA) is an exploratory data analysis technique for boolean relations based on lattice theory. Its main result is the existence of a dual order isomorphism between two set lattices induced by a binary relation between a set of objects and a set of attributes. Pairs of dually isomorphic sets of objects and attributes, called formal concepts, form a concept lattice, but actually model only a conjunctive mode of conceptualisation.

In this paper we augment this formalism in two ways: first we extend FCA to consider different modes of conceptualisation by changing the basic dual isomorphism in a modal-logic motivated way. This creates the three new types of concepts and lattices of extended FCA, viz., the lattice of neighbourhood of objects, that of attributes and the lattice of unrelatedness.

Second, we consider incidences with values in idempotent semirings - concretely the completed max-plus or schedule algebra $\overline{\mathbb{R}}_{\max ,+}$ - and focus on generalising FCA to try and replicate the modes of conceptualisation mentioned above.

To provide a concrete example of the use of these techniques, we analyse the performance of multi-class classifiers by conceptually analysing their confusion matrices.
\end{abstract}

Key words: Generalised Formal Concept Analysis, concept lattice, neighbourhood lattice, idempotent semiring, dioid, confusion matrix

\section{Introduction}

1.1. Formal Concept Analysis: a tool for interactive, exploratory data analysis

Formal Concept Analysis (FCA) 15] is a data exploration and visualisation technique rooted in Lattice Theory [4]. The basic objects of Formal Concept Analysis are formal contexts and concept lattices. A formal context $(G, M, I)$

\footnotetext{
* Corresponding author

Email addresses: fva@tsc.uc3m.es (Francisco J. Valverde-Albacete), carmen@tsc.uc3m.es (Carmen Peláez-Moreno)
} 
15] consists of a set of objects $G$, a set of attributes $M$ and an incidence $I$, a binary relation describing which objects show which attributes. For all sets of objects $A \subseteq G$, call object polar (with respect to $(G, M, I)$ ) the map $A^{\prime}=\{m \in M \mid \forall g \in A g I m\}$. Similarly, for a set of attributes $B \subseteq M$ call attribute polar the map $B^{\prime}=\{g \in G \mid \forall m \in B g I m\}$. Pairs $(A, B)$ such that $A^{\prime}=B$ and $B^{\prime}=A$ are the formal concepts of context $(G, M, I)$. Call $\mathfrak{B}(G, M, I)$ the set of all such concepts. If we define the order $\left(A_{1}, B_{1}\right) \leq$ $\left(A_{2}, B_{2}\right) \Leftrightarrow A_{1} \subseteq A_{2} \Leftrightarrow B_{1} \supseteq B_{2}$, then we can state:

Theorem 1.1. (Basic theorem of Concept Lattices, [15]) The set of concepts carries a complete lattice structure $\underline{\mathfrak{B}}(G, M, I) \equiv\langle\mathfrak{B}(G, M, I), \leq\rangle$, with and supremum given by

$$
\bigwedge_{t \in T}\left(A_{t}, B_{t}\right)=\left(\bigcap_{t \in T} A_{t},\left[\left[\bigcup_{t \in T} B_{t}\right]^{\prime}\right]^{\prime}\right) \bigvee_{t \in T}\left(A_{t}, B_{t}\right)=\left(\left[\left[\bigcup_{t \in T} A_{t}\right]^{\prime}\right]^{\prime}, \bigcap_{t \in T} B_{t}\right)
$$

Conversely, a complete lattice $V$ is isomorphic to $\underline{\mathfrak{B}}(G, M, I)$ if and only if there are mappings $\gamma: G \rightarrow V$ and $\mu: M \rightarrow V$ such that $\gamma(G)$ is join-dense in $V$, $\mu(M)$ is meet-dense in $V$, and $g I m$ is equivalent to $\gamma(g) \leq \mu(m)$ for all $g \in G$ and all $m \in M$.

As a data-exploration tool, a concept lattice is a device to describe the generalisation order between pairs of object and attribute sets, e.g. the (formal) concepts. What we are describing here are conjunctive units of conceptualisation 37. as dictated by the incidence $I$, the only source of information about the relationship of objects and attributes, that is $(A, B)$ is a formal concept for the context $(G, M, I)$ only because $I$ says so. If small, such lattices can be easily visualised, interpreted and navigated - and to some extent manipulated - by means of their Hasse diagrams. Indeed, this is what the Toscana and ToscanaJ data-exploration suites were designed for [2].

This paper is first and foremost an attempt at further broadening the scope of Formal Concept Analysis to make it a more generic tool for data mining purposes, and in this motivating section we present the directions in which we believe the theory should be further augmented.

\subsection{The question of alternative modes of conceptualisation}

It is widely acknowledged that data analysis processes bear more fruitful results when many different tools are offered to the practitioner. Since formal concepts only consider the case when all objects in the extent are incident with all attributes in the intent, it is advisable to ponder whether there are any other modes of conceptualisation apart from the conjunctive mode of formal concepts. As formal concepts are ordered in concept lattices, the question arises whether other types of "concepts" will also be ordered as lattices, and if so, what will their properties be. 
Table 1: Modal operators over a relation $I$ and its complement $I$ for sets of objects $A \subseteq G$ and attributes $B \subseteq M$.

\begin{tabular}{|c|c|}
\hline $\begin{array}{c}\text { possibility operator over } G \\
A_{I}^{\exists}=\{m \in M \mid(\exists g \in G)[g \in A \wedge g I m]\}\end{array}$ & $\begin{array}{c}\text { sufficiency operator over } G \\
\text { necessity operator over } M\end{array}$ \\
$A_{I}^{+}=\{m \in M \mid(\forall g \in G)[g \in A \Rightarrow g I m)\}$ \\
${ }_{I}^{\forall} B=\{g \in G \mid(\forall m \in M)[g I m \Rightarrow m \in B)\}$ & sufficiency operator over $M$ \\
\hline dual sufficiency operator over $G$ & ${ }_{I}^{+} B=\{g \in G \mid(\forall m \in M)[m \in B \Rightarrow g I m)\}$ \\
$A_{I}^{-}=\{m \in M \mid(\exists g \in G)[g \notin A \wedge g \mp m]\}$ & necessity operator over $G$ \\
dual sufficiency operator over $M$ & $A_{I}^{\forall}=\{m \in M \mid(\forall g \in G)[g I m \Rightarrow g \in A)\}$ \\
${ }_{I} B=\{g \in G \mid(\exists m \in M)[m \notin B \wedge g \mp m]\}$ & possibility operator over $M$ \\
\hline
\end{tabular}

One alternative conceptualisation is triadic FCA, proposed by Wille [36] and developed by Groh and Wille [20]. They consider a third set of "conditions" and carry out essentially the same set of operations on them, changing the double product set incidences belong to, into a triple product set. Yet another way of exploring alternate modes of conceptualisation is to relax the requisite on the equation defining the concepts. We thus arrive at semiconcepts and protoconcepts. These are crucial extensions that we will not pursue here.

Rather, we would like to reuse the results of standard FCA as much as possible, yet investigate new modes of conceptualisation. With such a purpose in mind, consider Table 1 listing the modal operators afforded by a Boolean matrix $I \in 2^{G \times M}$ over a set of objects $A \in 2^{G}$ and dually over sets of attributes $B \in 2^{M}$ (32, def. 3.8.2], 11]). FCA adepts will recognise the extent and intent polars in the sufficiency operators for a relation in the upper right quadrant, $A_{I}^{+}=A^{\prime},{ }_{I}^{+} B=B^{\prime}$, and their closure operators, ${ }_{I}^{+}\left(A_{I}^{+}\right)=A^{\prime \prime},\left({ }_{I}^{+} B\right)_{I}^{+}=B^{\prime \prime}$.

Perhaps less known is that the pairs of operators in the first and second rows of Table 1 define the neighbourhood lattices of objects. For a formal context $(G, M, I)$ define the span of a set of objects $A_{I}^{\exists}$ as the set of attributes related to some object $g \in A$. Similarly, define the content of a set of attributes ${ }_{I}^{\forall} B$ as the set of objects which can be completely described by the attributes in $B$. Next consider the set of neighbour pairs, $(A, B) \in \mathfrak{N}^{G}(G, M, I)$ such that $A_{I}^{\exists}=B \Leftrightarrow A={ }_{I}^{\forall} B$, where the systems of spans and contents are now order isomorphic: if $\left(A_{1}, B_{1}\right),\left(A_{2}, B_{2}\right)$ are neighbour pairs, $\left(A_{1}, B_{1}\right) \leq\left(A_{2}, B_{2}\right) \Longleftrightarrow$ $A_{1} \leq A_{2} \Longleftrightarrow B_{1} \leq B_{2}$. Then we can state the:

Theorem 1.2. (Basic theorem of Neighbourhood Lattices, [10]) $\mathfrak{N}^{G}(G, M, I)$ carries a complete lattice structure $\underline{\mathfrak{N}}^{G}(G, M, I)=\left\langle\mathfrak{N}^{G}(G, M, I), \leq\right\rangle$ in which infimum and supremum are given by

$$
\bigwedge_{t \in T}\left(A_{t}, B_{t}\right)=\left(\bigcap_{t \in T} A_{t},\left[{ }_{I}^{\forall}\left[\bigcap_{t \in T} B_{t}\right]\right]_{I}^{\exists}\right) \bigvee_{t \in T}\left(A_{t}, B_{t}\right)=\left(_{I}^{\forall}\left[\left[\bigcup_{t \in T} A_{t}\right]_{I}^{\exists}\right], \bigcup_{t \in T} B_{t}\right)
$$


Conversely, a complete lattice $V$ is isomorphic to $\underline{\mathfrak{N}}^{G}(G, M, I)$ if and only if there are mapping $\sigma: G \rightarrow V$ and $\kappa: M \rightarrow \bar{V}$ such that $\sigma(G) \cup\{0\}$ is join-dense in $V$ and $\kappa(M) \cup\{1\}$ is meet-dense in $V$ and $g I m$ is equivalent to $\sigma(g) \not \kappa(m)$ for all $g \in G$ and all $m \in M$.

This result shows that $\underline{\mathfrak{N}}^{G}(G, M, I)$ is isomorphic to $\underline{\mathfrak{B}}(G, M, \mp)$ [1] -where $\Psi$ is the complement of $I$-but the drawing and interpretation in terms of neighbourhood pairs must differ.

Of course, Neighbourhood and Concept Lattices are well-known in Galois connection theory, where they receive the name of axialities and polarities, respectively [13, 12]. The difference being actually made evident in these names is, on the one hand, an order isomorphism between an interior system of subsets of $G$ and a closure system of subsets of $M$ in the former case, and a dual order isomorphism between two closure systems of subsets of $G$ and $M$ in the latter case. This is, of course extendible, as explicitly done in [13], for one more order isomorphism between a closure system and an interior system of subsets of $G$ and $M$ respectively and yet another dual order isomorphism between two interior systems of subsets of $G$ and $M$.

Given the existence of such closure/kernel pairs, in this paper we also set out to extend FCA by creating analogues of its construction and representation results for these operators.

\subsection{The question of modelling degrees of incidence}

FCA can only represent the presence or absence of an association between particular objects and attributes. We may call this full and null incidence respectively. For some data domains, this can be too coarse an encoding.

Standard FCA of numerical domains can be improved by the introduction of scales that model sampling configurations of graded associations between objects and attributes 15, §. 1.3-4]. For instance one could conceive the continuum of people height in terms of three values tall, medium or short, and impose a value structure whereby somebody who is of height=medium can also be considered of height=short, i.e. make a one dimensional ordinal scale of cardinal $n=3[15$, p. 42]. Since the process of scaling needs a highly competent human expert and is ever prone to sampling and modelling errors, another crucial question for data mining applications based in the FCA paradigm is: are there similar operators to those described in subsection 1.2 on typical domains for data mining like $\mathbb{N}_{0}$ or $\mathbb{R}_{0}^{+}$or the probability interval $[0,1]$ ?

Burusco and Fuentes-González [5] proposed a first version of FCA over incidences with values in a fuzzy domain. This was later polished and completed developing into a well-established alternative to standard Formal Concept Analysis, Fuzzy (Formal) Concept Analysis in [27, 3, 22. However, the constraint that the values of the incidence belong to a fuzzy domain seems a bit too stringent: what is a good fuzzy domain for an attribute that might take negative

\footnotetext{
${ }^{1}$ Our names for the neighbour pair-creating functions to avoid those already taken by Formal Concept Analysis.
} 
and positive values like temperature? Again the data explorer should resort to scaling and this introduces a degree of subjectivity in the mining process that requires expert manipulation.

Yet another path towards generalising Formal Concept Analysis was explored in $\mathcal{K}$-Formal Concept Analysis [33, 35], an analogue where incidences take values in idempotent, reflexive semifields - closely related to residuated lattices - being structures in which the boolean semiring is embedded.

In this paper we also investigate how the construction leading to the pair of dually order-isomorphic lattices of $\mathcal{K}$-Formal Concept Analysis can be extended to obtain the other types of Galois Connections mentioned in Section 1.2 providing a fuller set of tools to interpret data [34].

\subsection{Reading guide}

To investigate the question of extending FCA with alternate conceptualisation modes, we adopt the wider setting of Galois connections between arbitrary orders (Section 2.1.1), provide a naming convention for all types of connections between two orders (Section 2.1.2) and finally review all Galois connections induced by a binary incidence (Section 2.1.3).

Afterwards, we first review idempotent semirings and semimodules - idempotent analogues of vector spaces - in Section 2.2 to investigate the question of generalising the domain over which incidences may take values; then, we reformulate in Section 2.3 the construction of Galois connections in idempotent semimodules to obtain the extension of FCA over incidences with values in an idempotent semifield, extended $\mathcal{K}$-Formal Concept Analysis (Section 2.4).

We provide an application instance by using the newly developed modes of conceptualisation for the analysis of confusion matrices of human speech recognition in Section 3 We end with a summary of contributions and a discussion including the relation between Fuzzy- and $\mathcal{K}$-Formal Concept Analysis in Section 4

\section{Theory and Calculations}

2.1. Extending Formal Concept Analysis: broadening conceptualisation modes

\subsubsection{Residuated maps, adjunctions and Galois connections}

Let $\mathcal{P}=\left\langle P, \leq_{\mathcal{P}}\right\rangle$ and $\mathcal{Q}=\left\langle Q, \leq_{\mathcal{Q}}\right\rangle$ be partially ordered sets. We have:

- A map $f: P \rightarrow Q$ is residuated if inverse images of principal (order) ideals of $Q$ under $f$ are again principal ideals. Its residual map or simply residual, $f^{\#}: Q \rightarrow P$ is $f^{\#}(q)=\max \left\{p \in P \mid f(p) \leq_{\mathcal{Q}} q\right\}$.

- A map $g: Q \rightarrow P$ is dually residuated if the inverse images of principal dual (order) ideals under $g$ are again dual ideals. Its dual residual map or simply dual residual, $g^{\mathrm{b}}: P \rightarrow Q$ is $g^{\mathrm{b}}(p)=\min \left\{q \in Q \mid p \leq_{\mathcal{P}} g(q)\right\}$. 
This duality of concepts is fortunately simplified by a well-known theorem stating that residual maps are dually residuated, while dual residual maps are residuated, hence we may maintain only the two notions of residuated maps and their residuals.

In fact, the two notions are so entwined that we give a name to them: an adjoint pair of maps $(\lambda, \rho)$ is a pair $(\lambda: P \rightarrow Q, \rho: Q \rightarrow P)$ between two ordered sets such that $\forall p \in P, q \in Q, p \leq_{\mathcal{P}} \rho(q) \Longleftrightarrow \lambda(p) \leq_{\mathcal{Q}} q$, equivalently, $p \leq_{\mathcal{P}} \rho(\lambda(p))$ and $\lambda(\rho(q)) \leq_{\mathcal{Q}} q$.

If the order relation is actually partial the lower or left adjoint, $\lambda$ is uniquely determined by its right or upper adjoint, $\rho$, and conversely 12, $\$ 1.1]$. The characterization theorem for adjoint maps [12, p. 7] states that $(\lambda, \rho)$ are adjoint if and only if, $\lambda$ is residuated with residual $\rho$, or equivalently, $\rho$ is dually residuated with $\lambda$ its dual residual.

Now consider the orders $\mathcal{P}=\left\langle P, \leq_{\mathcal{P}}\right\rangle$ and $\mathcal{Q}=\left\langle Q, \leq_{\mathcal{Q}}\right\rangle$ and their order duals $\mathcal{P}^{\mathrm{d}}=\left\langle P, \geq_{\mathcal{P}}\right\rangle$ and $\mathcal{Q}^{\mathrm{d}}=\left\langle Q, \geq_{\mathcal{Q}}\right\rangle$, to obtain two adjoint and two dually adjoint pairs:

Definition 1. (Different types of Galois connections and adjunctions)

1. $(\lambda, \rho)$ is a (Galois) adjunction on the left, and we write $(\lambda, \rho): \mathcal{P} \leftrightharpoons \mathcal{Q}$ iif: $\forall p \in P, q \in Q \quad \lambda(p) \leq_{\mathcal{Q}} q \Leftrightarrow p \leq_{\mathcal{P}} \rho(q)$, that is, the functions are covariant, and we say that $\lambda$ is the lower or left adjoint while $\rho$ is the upper or right adjoint

2. $(\rho, \lambda): \mathcal{P} \rightleftharpoons \mathcal{Q}$ is a (Galois) adjunction on the right iff: $\forall p \in P, q \in$ $Q \quad \rho(p) \geq_{\mathcal{Q}} q \Leftrightarrow p \leq_{\mathcal{P}} \lambda(q)$, both functions are covariant, $\rho$ is the upper adjoint, and $\lambda$ the lower adjoint.

3. $(\varphi, \psi)$ is a Galois Connection proper, of two dual adjoints $(\varphi, \psi): \mathcal{P} \leftrightharpoons \mathcal{Q}$ iff: $\forall p \in P, q \in Q \quad \varphi(p) \geq_{\mathcal{Q}} q \Leftrightarrow p \leq_{\mathcal{P}} \psi(q)$, that is, both functions are contravariant. For that reason they are sometimes named contravariant or symmetric adjunctions on the right. Note that $(\psi, \varphi)$ is also a TYPE OI Galois connection.

4. $\left(\triangle, \triangle^{\prime}\right)$ is a co-Galois connection, of dual adjoints $\left(\triangle, \triangle^{\prime}\right): \mathcal{P} \neg \mathcal{Q}$ if: $\forall p \in P, q \in Q \quad \triangle(p) \leq_{\mathcal{Q}} q \Leftrightarrow p \geq_{\mathcal{P}} \triangle^{\prime}(q)$, that is, both functions are contravariant. For that reason they are sometimes named contravariant or symmetric adjunctions on the left. $\left(\triangle^{\prime}, \triangle\right)$ is also a co-Galois connection.

Table 2 summarises the main properties of all types of Galois connections. Furthermore, as a sort of graphical summary, we introduce the diagram to the upper left-hand corner of figure 1 as the pattern that carries the structures described in 12, $§ 1.2]$. We illustrate how to read it with the diagram at the top left, which has:

- A closure system, $\rho(Q)=\bar{P}$, the closure range of the right adjoint (see below).

- An interior system, $\lambda(P)=\underline{Q}$, the kernel range of the left adjoint (see below). 
Table 2: Summary of Galois connections and their properties, for $P, Q$ posets.

\begin{tabular}{||c||c||}
\hline \hline Left Adjunction: $(\lambda, \rho): \mathcal{P} \leftrightharpoons \mathcal{Q}$ & Galois connection: $(\varphi, \psi): \mathcal{P} \leftrightharpoons \mathcal{Q}$ \\
\hline \hline$\forall p \in P, q \in Q \quad \lambda(p) \leq \mathcal{Q} q \Leftrightarrow p \leq \mathcal{P} \rho(q)$ & $\forall p \in P, q \in Q \quad \varphi(p) \geq_{\mathcal{Q}} q \Leftrightarrow p \leq \mathcal{P} \psi(q)$ \\
$I_{\mathcal{P}} \leq \rho \circ \lambda$ and $I_{\mathcal{Q}} \geq \lambda \circ \rho$ & $I_{\mathcal{P}} \leq \psi \circ \varphi$ and $I_{\mathcal{Q}} \leq \varphi \circ \psi$ \\
$\lambda=\lambda \circ \rho \circ \lambda$ and $\rho=\rho \circ \lambda \circ \rho$ & $\varphi=\varphi \circ \psi \circ \varphi$ and $\psi=\psi \circ \varphi \circ \psi$ \\
$\lambda$ monotone, residuated & $\varphi$ antitone \\
$\rho$ monotone, residual & $\psi$ antitone \\
$\lambda$ join-preserving, $\rho$ meet-preserving & $\varphi$ join-inverting, $\psi$ join-inverting \\
\hline \hline co-Galois connection: $\left(\triangle, \triangle^{\prime}\right): \mathcal{P} \nabla_{\mathcal{Q}}$ & Right Adjunction: $(\rho, \lambda): \mathcal{P} \rightleftharpoons \mathcal{Q}$ \\
\hline \hline$\forall p \in P, q \in Q \quad \triangle(p) \leq \mathcal{Q} q \Leftrightarrow p \geq \mathcal{P} \triangle^{\prime}(q)$ & $\forall p \in P, q \in Q \quad \rho(p) \geq \mathcal{Q} q \Leftrightarrow p \leq \mathcal{P}(q)$ \\
$I_{\mathcal{P}} \geq \triangle^{\prime} \circ \triangle$ and $I_{\mathcal{Q}} \geq \triangle^{\prime} \circ \triangle^{\prime}$ & $I_{\mathcal{P}} \geq \lambda \circ \rho$ and $I_{\mathcal{Q}} \leq \rho \circ \lambda$ \\
$\triangle=\triangle \circ \triangle^{\prime} \circ \triangle$ and $\triangle^{\prime}=\triangle^{\prime} \circ \triangle^{\prime} \circ \triangle^{\prime}$ & $\rho=\rho \circ \lambda \circ \rho$ and $\lambda=\lambda \circ \rho \circ \lambda$ \\
$\triangle$ antitone & $\rho$ monotone, residual \\
$\triangle$ antitone & $\lambda$ monotone, residuated \\
$\triangle$ meet-inverting, $\triangle^{\prime}$ meet-inverting & $\rho$ meet-preserving, $\lambda$ join-preserving \\
\hline \hline
\end{tabular}

- A closure function 11, 8, suggest "closure operator" $\gamma_{\mathcal{P}}=\rho \circ \lambda \geq_{\mathcal{P}} I_{\mathcal{P}}$, from $P$ to the closure range $\bar{P}=\rho(Q)$, with adjoint inclusion map $\hookrightarrow \mathcal{P}$, where $I_{\mathcal{P}}$ denotes the identity over $P$.

- A kernel function [11, also "interior operator", "kernel operator"] $\kappa_{\mathcal{P}}=$ $\lambda \circ \rho \leq_{\mathcal{Q}} I_{\mathcal{Q}}$, from $Q$ to the range of $\underline{Q}=\lambda(P)$, with adjoint inclusion map $\hookrightarrow_{\mathcal{Q}}$, where $I_{\mathcal{Q}}$ denotes the identity over $Q$.

- a perfect adjunction $(\tilde{\lambda}, \tilde{\rho}): \bar{P} \leftrightharpoons \underline{Q}$, that is, an order isomorphism between the closure and kernel ranges $\bar{P}$ and $\underline{Q}$.

Compare the mathematical objects above with those in a Galois connection proper seen in the top right of figure 11 the ranges are both closure systems and both compositions closure operators due to the dualisation of the second set (we write $\gamma_{\mathcal{Q}}$ for the new closure operator), resulting in the well-known perfect Galois connection, $(\tilde{\varphi}, \tilde{\psi}): \overline{\mathcal{P}} \sqcup \overline{\mathcal{Q}}$, the pair of dual order-isomorphic closure ranges lying at the heart of Formal Concept Analysis. The diagrams in the bottom left and right of figure 1 show analogue structures for co-Galois connections and right adjunctions respectively.

The different monotonicity conditions account for different properties of the adjoint maps [32, prop. 3.6.8]:

- if $(\lambda, \rho)$ form a left adjunction, then $\lambda$ is residuated, preserves existing least upper bounds (for lattices, joins) and $\rho$ preserves existing greatest lower bounds (for lattices, meets). 
- if $(\varphi, \psi)$ form a Galois connection, then both $\varphi$ and $\psi$ invert existing least upper bounds (for lattices, they transform joins into meets).

- if $(\rho, \lambda)$ form a right Galois connection, then $\rho$ preserves existing greatest lower bounds (meets for lattices) and $\lambda$ is residuated, preserves existing least upper bounds (joins for lattices).

- if $\left(\triangle, \triangle^{\prime}\right)$ form a co-Galois connection, then both $\triangle$ an $\triangle^{\prime}$ invert existing greatest lower bounds (for lattices, they transform meets into joins).

See 12 for a revision of the genesis and importance of Galois Connections and adjunctions, as well as a discussion of the different notation and nomenclatures for these concepts. 13] is an early tutorial with mathematical applications in mind.

\subsubsection{A proposal for a naming scheme for Galois connections}

We would now like to put forward a naming scheme to make the naming of Galois connections/adjunctions somewhat more homogeneous. We have related these names to the original ones by annotating figure 1 with them. Our naming scheme stresses the compositions with order- and dual order-isomorphisms, or anti-isomorphisms:

- We take the TYPE Oo Galois connection to be a basic adjunction composed with an even number of anti-isomorphism on the domain and range orders.

- To obtain a TYPE OI Galois connection compose a basic adjunction with an odd number of anti-isomorphism on the range.

- To get a a TYPE IO Galois connection we compose a basic adjunction with an odd number of anti-isomorphisms on the domain.

- Finally, a TYPE II Galois connection, is a basic adjunction with an odd number of anti-isomorphisms composed on both the domain and range.

\subsubsection{Extended conceptual analysis of binary incidences}

Let $I \in 2^{G \times M}$ be a concrete binary incidence. Obtaining the four types of connection induced by it is as easy as instantiating the abstract algebra constructions above considering the concrete order $2 P:=2^{G}$ and $Q:=2^{M}$, the power sets of a set of objects $G$ and a set of attributes $M$ with set inclusion order denoted by $\leq_{G}, \leq_{M}$.

Definition 2. [Broad-sense concept lattices 13, 10, 11, 15, , adapted]

- The TYPE OO Galois connection, the left adjunction $\left[(\cdot)_{I}^{\exists},{ }_{I}^{\forall}(\cdot)\right]: 2^{G} \leftrightharpoons 2^{M}$, generates the neighbourhood lattice of objects $\underline{\mathfrak{N}}^{G}(G, M, I)$.

\footnotetext{
${ }^{2}$ Here and elsewhere in this paper we use ": =", to be read "becomes", as an operation to pass from an abstract structure to a more concrete one.
} 
- The TYPe OI Galois connection proper $\left[(\cdot)_{I}^{+},{ }_{I}^{+}(\cdot)\right]: 2^{G} \sqcup 2^{M}$, generates the lattice at the heart of FCA $\underline{\mathfrak{B}}(G, M, I)$.

- The TYPe IO co-Galois connection $\left[(\cdot)_{I}^{-},{ }_{I}^{-}(\cdot)\right]: 2^{G} \nabla 2^{M}$, generates the lattice of unrelatedness $\underline{\mathfrak{B}}^{U}(G, M, I)$.

- The TYPE II Galois connection—right adjunction- $\left[(\cdot)_{I}^{\forall},{ }_{I}^{\exists}(\cdot)\right]: 2^{G} \rightleftharpoons 2^{M}$, generates the neighbourhood lattice of attributes $\underline{\mathfrak{N}}^{M}(G, M, I)$.

Note that the theorems describing these lattices are "for free": essentially concrete instantiations of those results in Section 2.1.1 This is graphically stated in figure 2

By the universal representation capabilities of the Basic Theorem of Concept Lattices, we know that broad-sense concept lattices are some sort of concept lattice from a TYPE OI connection. A very desirable feature of our extension would be that the newly introduced conceptualisation modes could be reduced to standard FCA techniques and algorithms. We must therefore look for formal contexts systematically related to $(G, M, I)$, say $\mathbb{K}^{\prime}$, whose standard concept lattices $\underline{\mathfrak{B}}\left(\mathbb{K}^{\prime}\right)$ represents a broad-sense concept lattice.

The necessary properties, summarised in Table 2 in universal form, follow:

Proposition 2.1. [10, Eqs. 2.8-2.11] $(\cdot)_{I}^{\exists}: 2^{G} \rightarrow 2^{M}$ preserves set unions. $(\cdot)_{I}^{\forall}: 2^{G} \rightarrow 2^{M}$ preserves set intersections. $(\cdot)_{I}^{+}: 2^{G} \rightarrow 2^{M}$ transforms set unions into intersections. $(\cdot)_{I}^{-}: 2^{G} \rightarrow 2^{M}$ transforms set intersection into unions.

Call $Y^{\mathbf{c}}=G \backslash Y$ the set complement of $Y, \bar{g}=\{g\}^{\mathbf{c}}$ and $\bar{m}=\{m\}^{\mathbf{c}}$. Then $(\cdot)_{I}^{\exists}$ and $(\cdot)_{I}^{+}$are defined by their action on singleton sets while $(\cdot)_{I}^{\forall}$ and $(\cdot)_{I}^{-}$ are defined by their action on the complementaries of singletons - and also for their (dually) adjoint maps, respectively.

Corollary 2.2. [10, Eqs. 2.6-2.7] For all $g \in G, m \in M$

$$
g_{I}^{\exists}=g_{I}^{+} \quad \bar{g}_{I}^{\forall}=\bar{g}_{I}^{-} \quad{ }_{I}^{\forall} m={ }_{I}^{+} m \quad{ }_{I}^{\exists} \bar{m}={ }_{I}^{-} \bar{m}
$$

Recall that the complement of a binary relation is $I=G \times M \backslash I$, its transpose or converse is $I^{\mathrm{T}}=\left\{\left(m_{j}, g_{i}\right) \in M \times G \mid\left(g_{i}, m_{j}\right) \in I\right\}$. It is folklore that the operators stand in a further duality, since $X^{c}=\bigcap_{g \in X} \bar{g}$ :

Proposition 2.3. [1], Eqs. 2.12] For all $Y \in 2^{G}, X \in 2^{M}$

$$
Y_{I}^{+}=\left(Y^{\mathbf{c}}\right)_{I}^{\forall} \quad Y_{I}^{-}=\left(Y^{\mathbf{c}}\right)_{I}^{\exists} \quad{ }_{I}^{+} X={ }_{I}^{\forall}\left(X^{\mathbf{c}}\right) \quad{ }_{I}^{-} X={ }_{I}^{\exists}\left(X^{\mathbf{c}}\right)
$$

Furthermore, in spite of the many maps needed to build up the connections, half of them can be built by transposing the incidence from the other maps:

Proposition 2.4. ${ }_{I}^{\exists}(\cdot): 2^{M} \rightarrow 2^{G}$ can be obtained as ${ }_{I}^{\exists}(\cdot)=(\cdot)_{I^{\mathrm{T}}}^{\exists}$. And similarly for ${ }_{I}^{\forall}(\cdot): 2^{M} \rightarrow 2^{G}$ with ${ }_{I}^{\forall}(\cdot)=(\cdot)_{I^{\mathrm{T}}}^{\forall} ;$ for ${ }_{I}^{+}(\cdot): 2^{M} \rightarrow 2^{G}$ with $I_{I}^{+}(\cdot)=(\cdot)_{I^{\mathrm{T}}}^{+}$ and for ${ }_{I}^{-}(\cdot): 2^{M} \rightarrow 2^{G}$ with ${ }_{I}^{-}(\cdot)=(\cdot)_{I^{\mathrm{T}}}^{-}$. 
Proof. In fact, this is the whole point of this positional-modal notation: flipping the side of the relation entails just using its converse.

Call $G^{-}=\{\bar{g} \mid g \in G\}, M^{-}=\{\bar{m} \mid m \in M\}$-which are not the complements of $G, M$ but their sets of element-wise complements. Then

Corollary 2.5. The broad-sence concept lattices induced by a binary relation can be represented as the (standard) Concept Lattices of as many formal contexts derived from $(G, M, I)$ by the following operations:

- The broad-sense concept lattice of $\left[(\cdot)_{I}^{\exists},{ }_{I}^{\forall}(\cdot)\right]: 2^{G} \leftrightharpoons 2^{M}$ is the Concept Lattice $\underline{\mathfrak{B}}_{\mathrm{oO}}(G, M, I) \cong \underline{\mathfrak{N}}^{G}(G, M, I) \cong \underline{\mathfrak{B}}\left(G, M^{-}, I\right)$.

- The broad-sense concept lattice of $\left[(\cdot)_{I}^{+},{ }_{I}^{+}(\cdot)\right]: 2^{G} \sqcup 2^{M}$ is just its Concept Lattice $\underline{\mathfrak{B}}_{\mathrm{OI}}(G, M, I) \cong \underline{\mathfrak{B}}(G, M, I)$.

- The broad-sense concept lattice of $\left.(\cdot)_{I}^{-},{ }_{I}^{-}(\cdot)\right]: 2^{G} \neg 2^{M}$ is the Concept Lattice $\underline{\mathfrak{B}}_{\mathrm{IO}}(G, M, I) \cong \underline{\mathfrak{B}}^{U}(G, M, I) \cong \underline{\mathfrak{B}}\left(G^{-}, M^{-}, I\right)$

- The (broad-sense) concept lattice of $\left[(\cdot)_{I}^{\forall},{ }_{I}^{\exists}(\cdot)\right]: 2^{G} \rightleftharpoons 2^{M}$ is the Concept Lattice $\underline{\mathfrak{B}}_{\mathrm{II}}(G, M, I) \cong \underline{\mathfrak{N}}^{M}(G, M, I) \cong \underline{\mathfrak{B}}\left(G^{-}, M, \Psi\right)$

We illustrate in figure 2 the four types of connection in terms of standard Concept Lattices. An example of how to use them can be found in Section 3.1 .

\subsection{Idempotent semifields and semimodules}

To generalise Formal Concept Analysis to accept degrees of incidence first we introduce idempotent semifields (Section 2.2.1) and their completions (Section 2.2.2) necessary to study residuation in them (Section 2.2.3). Of course our real interest lies in vector spaces of semiring-valued sets (Section 2.2.4) and residuation in them (Section 2.2.5).

\subsubsection{Semirings, dioids and idempotent semifields}

A semiring $\mathcal{S}=\langle S, \oplus, \otimes, \epsilon, e\rangle$ is an algebra whose additive structure, $\langle S, \oplus, \epsilon\rangle$, is a commutative monoid and whose multiplicative structure, $\langle S \backslash\{\epsilon\}, \otimes, e\rangle$, is a monoid with multiplication distributing over addition from right and left and with additive neutral element absorbing for $\otimes$, i.e. $\forall a \in S, \epsilon \otimes a=\epsilon$.

Example 1. The following semirings are to be contrasted in the text:

1. The natural numbers with zero $\mathbb{N}_{0} \equiv\langle\mathbb{N} \cup\{0\},+, \times, 0,1\rangle$.

2. The integers $\mathbb{Z} \equiv\langle\mathbb{Z},+, \times, 0,1\rangle$.

3. The positive rationals with zero $\mathbb{Q}_{0}^{+} \equiv\left\langle\mathbb{Q}^{+} \cup\{0\},+, \times, 0,1\right\rangle$.

4. The Boolean lattice $\mathbb{B}=\langle\{0,1\}, \vee, \wedge, 0,1\rangle$.

5. The tropical semiring $\mathbb{N}_{\text {min },+} \equiv\langle\mathbb{N} \cup\{0, \infty\}$, min, $+, \infty, 0\rangle$.

6. The optimization algebra, $\mathbb{R}_{\min ,+}=\langle\mathbb{R} \cup\{\infty\}$, min, $+, \infty, 0\rangle$.

7. The schedule algebra $\mathbb{R}_{\max ,+}=\langle\mathbb{R} \cup\{-\infty\}$, max, $+,-\infty, 0\rangle$. 
Notice how the abstract notation for the zero and unit elements solves the problem of notation for semirings where 0 (the digit) acts as $e$ (the unit) as in the last three examples.

A well known result for semirings is that the multiplicative and additive structures are completely independent, what accounts for their abundance. These structures also have different importance in the classification of semirings, as shown below.

Regarding the multiplicative structure, left and right multiplications can be defined on any semiring $\mathcal{S}$

$$
\begin{array}{ll}
\mathrm{L}_{a}: K \rightarrow K & \mathrm{R}_{a}: K \rightarrow K \\
b \mapsto \mathrm{L}_{a}(b)=a b & b \mapsto \mathrm{R}_{a}(b)=b a
\end{array}
$$

A commutative semiring is one whose multiplicative structure is commutative. A multiplicatively idempotent semiring is one whose multiplicative structure is an idempotent semigroup, and a semifield one whose multiplicative structure $\langle S \backslash\{\epsilon\}, \otimes, e\rangle$ is a group, that is, there is an operation, ${ }^{-1}: S \backslash\{\epsilon\} \rightarrow S \backslash\{\epsilon\}$ such that $\forall a \in S, a \otimes a^{-1}=a^{-1} \otimes a=e$. For commutative semifields whose multiplicative structure is a commutative group we have $(a \otimes b)^{-1}=a^{-1} \otimes b^{-1}$.

Focusing on the additive structure, a semiring is cancellative if for $a, b, c \in S$ when $a \oplus b=a \oplus c$ then $b=c$. For instance, the integers and the natural numbers are cancellative, but the boolean and tropical semirings and the schedule algebra are not.

A ring is a cancellative semiring whose additive structure is that of a group, $\forall a \in S, \exists c \in S, a \oplus c=\epsilon$. A semiring is zerosumfree-or positive-if for $a, b \in S$ when $a \oplus b=\epsilon$ then $a=b=\epsilon$ : compared to a ring, zerosumfree semirings crucially lack additive inverses. In fact, rings are as "far away" as possible from zerosumfree semirings, the singleton zero element being the only semiring that is both. Note that $\mathbb{N}_{0}$ and $\mathbb{B}$ are zerosumfree whereas $\mathbb{Z}$ is not .

A semiring $\mathcal{D}$ is a dioi $\mathbb{3}$ or naturally-or canonically-ordered if for $a, b, c \in$ $D a \oplus c=b$ and $b \oplus d=a$ entails $a=b$. The order implied here is $a \preccurlyeq b$ iff $\exists c \in S, a \oplus c=b$. Moreover, the neutral element for the additive structure of semiring $\mathcal{D}$ is the infimum for this natural order, $\epsilon=\perp$. A transcendental result is that canonically-ordered semirings are zerosumfree [16, p. 32]. For instance the boolean semiring and the natural numbers are dioids in their usual orders. The schedule algebra is a dioid canonically-ordered as $\mathbb{R}$ and the tropical semiring has the dual order to $\mathbb{N}_{0}$.

An (additively) idempotent semiring $\mathcal{D}$ is a semiring whose addition is idempotent, $\forall a \in D, a \oplus a=a$, that is, whose additive structure $\langle D, \oplus, \epsilon\rangle$ is an idempotent semigroup. Idempotent semirings are all dioids, thus zerosumfree 16, p. 33]. Furthermore, the canonical order turns idempotent semirings into sup-semilattices $\langle D, \vee\rangle$ with least upper bound defined as $a \vee b=a \oplus b$. We now contrast two important classes of idempotent semirings.

\footnotetext{
${ }^{3}$ For "double monoid".
} 
- A doubly idempotent dioid is an idempotent semiring with an idempotent, commutative multiplicative structure [19, §.1.6.5].

- An idempotent semifield is an idempotent semiring $\mathcal{K}$ whose multiplicative structure $\left\langle K \backslash\{\epsilon\}, \otimes, e, \cdot^{-1}\right\rangle$ is a group.

Of the above examples, only $\mathbb{B}, \mathbb{R}_{\max ,+}$ and $\mathbb{N}_{\min ,+}$ are idempotent semirings, but $\mathbb{B}$ and $\mathbb{R}_{\max ,+}$ (with inverse $\cdot{ }^{-1}:=-$.) are also idempotent semifields. On the other hand, $\mathbb{B}$, and indeed all distributive lattices, are doubly idempotent dioids.

In an idempotent semifield the formula for the infimum of two elements was already given by Dedekind [12, §4.2]: the meet law is $a \wedge b=a \otimes(a \oplus b)^{-1} \otimes b$ thus idempotent semifields are lattices in their canonical order $\langle K, \vee, \wedge\rangle$.

\subsubsection{Complete semirings, semifields and blogs}

A semiring $\mathcal{S}$ is complete, if for any index set $I$ including the empty set, and any $\left\{a_{i}\right\}_{i \in I} \subseteq \mathcal{S}$ the (possibly infinite) summations $\sum_{i \in I} a_{i}$ are defined and the distributivity conditions: $\left(\sum_{i \in I} a_{i}\right) \otimes c=\sum_{i \in I}\left(a_{i} \otimes c\right)$ and $c \otimes\left(\sum_{i \in I} a_{i}\right)=$ $\sum_{i \in I}\left(c \otimes a_{i}\right)$, are satisfied. Note that for $c=e$ the above demand that infinite sums have a result.

A dioid $\mathcal{D}$ is complete, if it is further complete as a naturally ordered set $\langle D, \preceq\rangle$ and left $\left(\mathrm{L}_{a}\right)$ and right $\left(\mathrm{R}_{a}\right)$ multiplications are residuated.

A standard result in order theory [8] asserts that complete dioids, as complete join-semilattices with a bottom element, are already complete lattices $\langle K, \vee=$ $\oplus, \wedge, \perp=\epsilon, \top\rangle$. The top element $T$, the supremum in the canonical order, is the sum of all the elements in the dioid $\top=\sum_{a \in D} a$ so that $\top \oplus a=\top$. By the semiring axioms, however, we have: $T \otimes \epsilon=\epsilon$.

For complete idempotent semifields, the expression of the meet is given by Dedekind's formula. However, a canonically-ordered semifield $\mathcal{K} \neq\{\epsilon, e\}$ (that is, non-isomorphic to $\mathbb{B}$ ) cannot contain a top element $T$ (nor a bottom!) hence it cannot be a complete dioid [14, p. 160].

Example 2. In the schedule algebra $\mathbb{R}_{\max ,+}$ we have $a^{k}:=k \cdot a$ and therefore $e \oplus a^{k}:=\max (0, k \cdot a)$. Thus, for $a \geq 0, e \oplus a \oplus \ldots a^{k}=a^{k}:=k \cdot a$ and such summations converge, rendering $\mathbb{R}_{\max ,+}$ incomplete. The optimization algebra $\mathbb{R}_{\min ,+}$ is incomplete for similar reasons.

Next we summarize from [26, 29, 30, 31] how one may obtain from any (incomplete) idempotent semiring $\mathcal{D}$ a completion as follows:

\section{Construction 1. Canonical enlargement of a lattice-ordered group.}

1. For any lattice-ordered group $\mathcal{G}=\langle G, \preceq, \otimes\rangle$ : adjoin two elements $\perp$ and $\top$ to $G$ to obtain $\bar{G}=G \cup\{\perp, \top\}$ and extend the order to $\bar{G}$ as $\perp \preceq a \preceq \top, \forall a \in \bar{G}$. Then extend the product to two different operations, 
upper $\dot{\otimes}$ and lower $\otimes$ multiplications:

$$
\begin{aligned}
& a \otimes b= \begin{cases}\perp & \text { if } a, b \in G \cup\{\perp, \top\}, \text { with } a=\perp, \text { or } b=\perp . \\
\top & \text { if } a, b \in G \cup\{\top\}, \text { with } a=\top, \text { or } b=\top . \\
a \otimes b & \text { if } a, b \in G .\end{cases} \\
& a \dot{\otimes} b= \begin{cases}\top & \text { if } a, b \in G \cup\{\perp, \top\}, \text { with } a=\top, \text { or } b=\top . \\
\perp & \text { if } a, b \in G \cup\{\perp\}, \text { with } a=\perp, \text { or } b=\perp . \\
a \otimes b & \text { if } a, b \in G .\end{cases}
\end{aligned}
$$

to obtain the structure $\overline{\mathcal{G}}=\langle\bar{G}, \preceq, \dot{\otimes}, \otimes\rangle$, known as the canonical enlargement of $\mathcal{G}=\langle G, \preceq, \otimes\rangle$. In this structure, $\otimes$ and $\dot{\otimes}$ are associative and commutative over $\bar{G}$, as the original $\otimes$ was over $G$, and the isotonicity of the product with respect to the natural order extends to $\overline{\mathcal{G}}$. Furthermore, if $e$ is the unit element of $\langle G, \otimes\rangle$, it is similarly the unit of $\langle\bar{G}, \dot{\otimes}\rangle$ and $\langle\bar{G}, \otimes\rangle$.

2. The top completion of a dioid $\mathcal{D}$ is another dioid $\overline{\mathcal{D}}=\langle\bar{D}, \oplus, \otimes, \epsilon, e\rangle$ where: $\bar{D}=D \cup\{\top\}$ and in which $\otimes$ coincides with its definition above when $\mathcal{D}$ is considered as bearing a lattice-ordered (multiplicative semi-)group, $\perp=\epsilon$, and we extend $\oplus$ with the extra top-element:

$$
a \oplus b= \begin{cases}\top & \text { if } a=\top \text { or } b=\top . \\ a \oplus b, & \text { if } a, b \in D .\end{cases}
$$

3. Given an (incomplete) idempotent semifield $\mathcal{K}$, on its top enlargement as a dioid, $\overline{\mathcal{K}}$, we extend the notation for the inverse with the following conventions: $\epsilon^{-1}=\top, T^{-1}=\epsilon$.

In that way we have two related completed idempotent semifield structures:

- a complete lattice for the natural order $\langle\bar{K}, \preccurlyeq\rangle$, the one we have been focusing on, $\overline{\mathcal{K}}=\left\langle\bar{K}, \oplus=\vee, \otimes, \cdot{ }^{-1}, \perp, e\right\rangle$, and

- a complete lattice for the dual order, $\left\langle\bar{K}, \preccurlyeq^{\mathrm{d}}\right\rangle=\langle\bar{K}, \succcurlyeq\rangle, \overline{\mathcal{K}}^{\mathrm{d}}=\langle\bar{K}, \dot{\oplus}=$ $\left.\wedge, \dot{\otimes},{ }^{-1}, \top, e\right\rangle$ where the meet is defined (on $\overline{\mathcal{K}}$ ) by Dedekind's formula and the definition of $\dot{\otimes}$ follows equation (17).

Notice that the orders in $\overline{\mathcal{K}}$ and $\overline{\mathcal{K}}^{\mathrm{d}}$ are dual. Indeed, the dually-ordered completion of each is actually the other: it is easy to see that the meet in $\overline{\mathcal{K}}$ is the join in $\overline{\mathcal{K}}^{\mathrm{d}}$, and viceversa, by Dedekind's formula. This perfect Galois connection of TYPE OI is accomplished by the inversion $\left(\cdot{ }^{-1},{ }^{-1}\right): \overline{\mathcal{K}} \sqcup \overline{\mathcal{K}}^{\mathrm{d}}$. 
This double structure $\left(\overline{\mathcal{K}}, \overline{\mathcal{K}}^{\mathrm{d}}\right)=\left\langle\bar{K}, \oplus, \dot{\oplus}, \otimes, \dot{\otimes},{ }^{-1}, \perp, e, \top\right\rangle$ was first recorded as a blog, a bounded lattice-ordered group by Cuninghame-Green [7, §4.1], although the name did not catch. Its usefulness is evident, for instance in:

Proposition 2.6 (31], lemma 2.2). In the top enlargement $\left(\overline{\mathcal{K}}, \overline{\mathcal{K}}^{\mathrm{d}}\right)$ of any commutative semifield $\mathcal{K}$ we have the following De Morgan-like relations between additions, multiplications and inversion:

$$
\begin{array}{ll}
(a \oplus b)^{-1}=a^{-1} \dot{\oplus} b^{-1} & (a \dot{\oplus} b)^{-1}=a^{-1} \oplus b^{-1} \\
(a \otimes b)^{-1}=a^{-1} \dot{\otimes} b^{-1} & (a \dot{\otimes} b)^{-1}=a^{-1} \otimes b^{-1}
\end{array}
$$

Example 3. [Top completions of $\mathbb{R}_{\max ,+}$ and $\mathbb{R}_{\min ,+}$ ]

- The top completion of $\mathbb{R}_{\max ,+}$ is $\overline{\mathbb{R}}_{\max ,+}=\langle\mathbb{R} \cup\{ \pm \infty\}, \max ,+, \cdot-,-\infty, 0\rangle$, the completed Maxplus semifield.

- The top completion of $\mathbb{R}_{\min ,+}$ is $\overline{\mathbb{R}}_{\min ,+}=\langle\mathbb{R} \cup\{ \pm \infty\}, \min , \dot{+}, \cdot-, \infty, 0\rangle$ the completed Minplus semifield.

In this notation, due to Moreau [26], we have $-\infty+\infty=-\infty$ and $-\infty \dot{+} \infty=$ $\infty$, solving some issues in dealing with the incomplete semifields at a time.

Example 4. The lattice $\mathbb{B}$ can be embedded in any blog, by restricting the carrier set to $\{\perp, \top\}$ so that the boolean operations be implemented as $\oplus$ and $\otimes$ restricted to such set. The dually-ordered embedding is $\mathbb{B}^{\mathrm{d}}$, of course.

A richer structure, isomorphically embeddable in any blog [7, Theorem 4.5], is the 3-element blog $\mathbb{G}=\langle\{\perp, e, \top\}, \oplus, \dot{\oplus}, \otimes, \dot{\otimes}, \perp, e, \top\rangle$. This is also the only blog having a finite number of elements [7, Propositions 4.6-4.9].

\subsubsection{Residuation in dioids and idempotent semifields}

Since dioids are ordered sets, left and right residuated maps can be defined for the the left and right actions (multiplications) of equation 囵:

$$
a \backslash b=\mathrm{L}_{a}^{\#}(b)=\vee\{x \mid a \otimes x \preccurlyeq b\} \quad a / b=\mathrm{R}_{a}^{\#}(b)=\vee\{x \mid x \otimes a \preccurlyeq b\}
$$

A semiring is residuated if the left and right maps are residuated for all values of $a \in \mathcal{D}$. Hence complete dioids are residuated.

If the blog $\left(\overline{\mathcal{K}}, \overline{\mathcal{K}}^{\mathrm{d}}\right)$ is the completion of an idempotent semifield (see 2.2.2), its upper and lower residuals are:

$$
a \otimes b \preccurlyeq c \Leftrightarrow b \preccurlyeq a \backslash c \Leftrightarrow a \preccurlyeq c / b \quad a \dot{\otimes} b \preccurlyeq^{\mathrm{d}} c \Leftrightarrow b \preccurlyeq^{\mathrm{d}} a \backslash c \Leftrightarrow a \preccurlyeq^{\mathrm{d}} c / \dot{b}
$$

Again, blogs prove extremely useful because: 
Proposition 2.7. In the top enlargement $\left(\overline{\mathcal{K}}, \overline{\mathcal{K}}^{\mathrm{d}}\right)$ of any commutative semifield $\mathcal{K}$ residuals can be expressed in terms of the multiplications and inversion as

$$
\begin{aligned}
& a \backslash c=a^{-1} \dot{\otimes} c=\left(a \otimes c^{-1}\right)^{-1} \quad c / a=c \dot{\otimes} a^{-1}=\left(c^{-1} \otimes a\right)^{-1} \\
& a \dot{\backslash} c=a^{-1} \otimes c=\left(a \dot{\otimes} c^{-1}\right)^{-1} \quad c j a=c \otimes a^{-1}=\left(c^{-1} \dot{\otimes} a\right)^{-1}
\end{aligned}
$$

\subsubsection{Idempotent semimodules}

A semimodule over a semiring is defined in a similar way to a module over a ring [6, 18, 17]: a right $\mathcal{S}$-semimodule $\mathcal{X}$ over a semiring $\mathcal{S}=\left\langle S, \oplus, \otimes, \epsilon_{\mathcal{S}}, e_{\mathcal{S}}\right\rangle$ is an additive commutative monoid $\left\langle X, \oplus, \epsilon_{\mathcal{X}}\right\rangle$ endowed with a right action $(x, \lambda) \mapsto x \odot \lambda$ such that $\forall \lambda, \mu \in S, \quad x, x^{\prime} \in Y$. Following the convention of dropping the symbols for the scalar action and semiring multiplication we have:

$$
\begin{aligned}
x(\lambda \mu) & =(x \lambda) \mu & & x \epsilon_{S}=\epsilon_{\mathcal{X}} \\
\left(x \oplus x^{\prime}\right) \lambda & =x \lambda \oplus x^{\prime} \lambda & & x e_{S}=x
\end{aligned}
$$

Given $x, x^{\prime} \in X, \lambda \in S$ the left and right multiplications are $\mathrm{L}_{x}: S \rightarrow$ $X, \mathrm{~L}_{x}(\lambda)=x \lambda$ and $\mathrm{R}_{\lambda}: X \rightarrow X, \mathrm{R}_{\lambda}(x)=x \lambda$.

The definition of a left $\mathcal{S}$-semimodule $\mathcal{Y}$ follows the same pattern with the help of a left action, $(x, \lambda) \mapsto \lambda \odot x$ and similar axioms to those of (13). A $(\mathcal{R}, \mathcal{S})$-semimodule is a set $M$ endowed with left $\mathcal{R}$-semimodule and a right $\mathcal{S}$ semimodule structures, and a $(\mathcal{R}, \mathcal{S})$-bisemimodule a $(\mathcal{R}, \mathcal{S})$-semimodule such that the left and right actions commute.

Example 5. Each semiring, $\mathcal{S}$, is a left (right) semimodule over itself, with the semiring product as left (right) action. Therefore, it is a $(\mathcal{S}, \mathcal{S})$-bisemimodule over itself, because both actions commute by associativity. Such is the case for the Boolean, the max-plus and the min-plus bisemimodules.

A right (left) $\mathcal{D}$-moduloid is a right (left) semimodule over a dioid $\mathcal{D}$. Similarly we have $(\mathcal{S}, \mathcal{D})$-bimoduloids. We prefer "idempotent semimodules" to "idempotent moduloids". A right semimodule $\mathcal{X}$ over an idempotent semiring $\mathcal{D}$ inherits the idempotent law: $\forall x \in X, x \oplus x=x$, which induces a natural order on the semimodule: $\forall x, x^{\prime} \in X, x \preccurlyeq x^{\prime} \Longleftrightarrow x \oplus x^{\prime}=x^{\prime}$, whereby it becomes a $\vee$-semilattice, with $\epsilon_{\mathcal{X}}$ its minimum.

When $\mathcal{D}$ is a complete idempotent semiring, a right $\mathcal{D}$-semimodule $\mathcal{X}$ is complete (in its natural order) if it is complete as a naturally ordered set and its left and right multiplications are residuated. Trivially, it is also a complete lattice, with join and meet operations given by: $x \preccurlyeq x^{\prime} \Longleftrightarrow x \vee x^{\prime}=x^{\prime} \Longleftrightarrow$ $x \wedge x^{\prime}=x$.

Example 6 (Finite matrix semirings and semimodules). Let $\mathcal{S}$ be a semiring. $\mathcal{M}_{n}(\mathcal{S})=\left\langle S^{n \times n}, \oplus, \otimes, \mathcal{E}, I\right\rangle$ is the semiring of (square) matrices over $\mathcal{S}$ with $S^{n \times n}$ denoting the set of square matrices of order $n$ with entries in the semiring, matrix operations $(A \oplus B)_{i j}=A_{i j} \oplus B_{i j}, 0 \leq i, j \leq n$ and 
$(A \otimes B)_{i j}=\sum_{k=1}^{n} A_{i k} \otimes B_{k j}, 0 \leq i, j \leq n$, null matrix $\mathcal{E}, \mathcal{E}_{i j}=\epsilon, 0 \leq i, j \leq n$ and unit matrix $I, I_{i i}=e, 0 \leq i \leq n, I_{i j}=\epsilon, 0 \leq i, j \leq n, i \neq j$. If $\overline{\mathcal{K}}$ is a completed semifield, then matrix multiplications for conformant $A, B$ are:

$$
(A \otimes B)_{i j}=\sum_{k=1}^{n} A_{i k} \otimes B_{k j} \quad(A \dot{\otimes} B)_{i j}=\sum_{k=1}^{n} \bullet A_{i k} \dot{\otimes} B_{k j}
$$

Such semirings are not commutative in general even if $\mathcal{S}$ is, except for $\mathcal{M}_{1}(\mathcal{S})=$ $\mathcal{S}$. They are idempotent and complete if $\mathcal{S}$ is.

For $n, p \in \mathbb{N}$, the semimodule of finite matrices $\mathcal{M}_{n \times p}(\mathcal{S})=\left\langle S^{n \times p}, \oplus, \mathcal{E}\right\rangle$ is a $\left(\mathcal{M}_{n}(\mathcal{S}), \mathcal{M}_{p}(\mathcal{S})\right)$-bisemimodule, with matrix multiplication-like left and right actions and entry-wise addition. Special cases of it are the bisemimodules of column vectors $\mathcal{M}_{p \times 1}(\mathcal{S})$ and row vectors $\mathcal{M}_{1 \times n}(\mathcal{S})$.

In the following we systematically equate left (resp. right) $\mathcal{S}$-semimodules and row (resp. column) semimodules over $\mathcal{S}$.

In a semimodule $\mathcal{X}$ over a semifield $\mathcal{K}$ one can define and element-wise inversion operation $\cdot^{-1}: X \rightarrow X, x \mapsto x^{-1}$ such that $\left(x^{-1}\right)_{i}=x_{i}{ }^{-1}$. If the semifield is also a dioid, then the "inverse" semimodule is the order dual $\mathcal{X}^{-1} \cong\langle X, \preccurlyeq$ d $\rangle$.

Example 7. Semimodules over $\overline{\mathbb{R}}_{\max ,+}$ have inverses over $\overline{\mathbb{R}}_{\text {min },+}$ and vice versa. In particular $\overline{\mathbb{R}}_{\max ,+}^{-1}=\overline{\mathbb{R}}_{\min ,+}$.

\subsubsection{Residuation in idempotent semimodules}

Let $\mathcal{X}$ be a right semimodule over a complete, idempotent semiring $\mathcal{D}$. Given $x, x^{\prime} \in X, \lambda \in D$ the left and right residuals of the action are

$$
x \backslash x^{\prime}=\mathrm{L}_{x}^{\#}\left(x^{\prime}\right)=\vee\{\lambda \in D \mid x \lambda \preccurlyeq b\} \quad x^{\prime} / \lambda=\mathrm{R}_{\lambda}^{\#}\left(x^{\prime}\right)=\vee\left\{x \in X \mid x \lambda \preccurlyeq x^{\prime}\right\}
$$

with residuation law $\lambda \preccurlyeq x \backslash x^{\prime} \Leftrightarrow x \lambda \preccurlyeq x^{\prime} \Leftrightarrow x \preccurlyeq x^{\prime} / \lambda$. This extends naturally to left semimodules and bisemimodules. Calculation rules for residuation are specially straightforward in semimodules of completed idempotent semifields (see section 2.3.2).

There is a remarkable operation that changes the character of a semimodule while at the same time inverting its order by means of residuation: let $\mathcal{X}$ be a complete, idempotent right $\mathcal{D}$-semimodule, its opposite semimodule is the complete left $\mathcal{D}$-semimodule $\mathcal{X}^{\mathrm{op}}=\langle X, \stackrel{\mathrm{op}}{\oplus}, \stackrel{\mathrm{op}}{\rightarrow}\rangle$ with the same underlying set $X$, addition defined by $\left(x_{1}, x_{2}\right) \mapsto x_{1} \stackrel{\text { op }}{\oplus} x_{2}=x_{1} \wedge x_{2}$ where the infimum is for the natural order of $\mathcal{X}$, and left action $\lambda \stackrel{\text { op }}{\rightarrow} x=x / \lambda$. Consequently, the order of the opposite is the dual of the original order. For the opposite semimodule the residual definitions are:

$$
\begin{gathered}
\lambda \backslash x=\left(L_{\lambda}^{\mathcal{X}^{\mathrm{op}}}\right)^{\#}(x)=\bigwedge\left\{x^{\prime} \in X \mid x \preccurlyeq x^{\prime} / \lambda\right\}=x \cdot \lambda \\
x / x^{\prime}=\left(R_{x^{\prime}}^{\mathcal{X}^{\mathrm{op}}}\right)^{\#}(x)=\bigvee\left\{\lambda \in K \mid x \preccurlyeq x^{\prime} / \lambda\right\}=x \backslash x^{\prime}
\end{gathered}
$$


Note that we can define mutatis mutandis the opposite semimodule of a left $\mathcal{D}$ semimodule, $\mathcal{Y}$, with right action $y \stackrel{\text { op }}{\longleftarrow} \lambda=\lambda \backslash y$. Also, note that all dioids $\mathcal{D}$, as $(\mathcal{D}, \mathcal{D})$-bisemimodules, accept an opposite semiring, $\mathcal{D}^{\text {op }}$. And finally, that the operation of finding the opposite of a complete (left, right) $\mathcal{D}$-semimodule is an involution: $\left(\mathcal{X}^{\mathrm{op}}\right)^{\mathrm{op}}=\mathcal{X}$, since the first residual in (16) is in fact an involution. But note that inverses are not opposites (although they show the same order). It can be easily proven that opposition and inversion commute.

Example 8 (Opposite Boolean semifield). $\mathbb{B}^{\mathrm{op}}=\langle\underset{\mathrm{op}}{\{\mathbf{1}, \mathbf{0}\}} \stackrel{\mathrm{op}}{\oplus}, \stackrel{\mathrm{op}}{\odot}, \mathbf{1}, \mathbf{0}\rangle$ is also a complete bisemimodule where addition is the meet $v \oplus w=v \wedge w$. Consequently, its natural order is the inverse of the usual order, the additively neutral element is $\epsilon_{\mathbb{B}^{\circ}}=\mathbf{1}$, which is the bottom for the opposite natural order, and the action is the residual of the original action. In fact, the truth table for this connective is that of the logical conditional $\lambda \stackrel{\text { op }}{\rightarrow} x=x / \lambda=\lambda \backslash x=\lambda \rightarrow x$.

Example 9 (Opposite semifields to $\overline{\mathbb{R}}_{\max ,+}$ and $\overline{\mathbb{R}}_{\min ,+}$ ). As as right semimo-

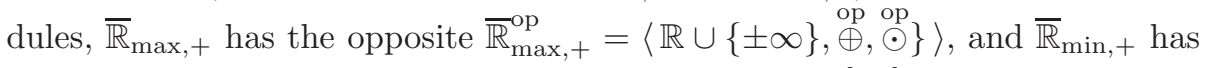
the opposite $\left.\overline{\mathbb{R}}_{\mathrm{min},+}^{\mathrm{op}}=\langle\mathbb{R} \cup\{ \pm \infty\}, \underset{\mathrm{op}}{\dot{\oplus}}, \dot{\odot}\}\right\rangle$, with $\underset{\text { op }}{\oplus}=\dot{\oplus}$ and $\lambda \stackrel{\text { op }}{\otimes} x=x / \lambda=$ $x \dot{\otimes} \lambda^{\circledast}$. Similarly $\underset{\text { op }}{\dot{\oplus}}=\underset{\text {. }}{\oplus} \underset{\text { op }}{\lambda \dot{\otimes}} x=x \dot{j} \lambda=x \otimes \lambda^{\circledast}$.

\subsection{Galois connections for incidences with values in idempotent semifields}

In this section we provide an easy way to build all possible Galois connections between two semimodules over complete idempotent semifields: first we introduce Galois connections of type TYPE OI between idempotent semimodules as basic building blocks (Section 2.3.1). Since Sections 2.1.2 and 2.1.3 highlighted the role of anti-isomorphisms in the definition of the different types of Galois connection, we next introduce conjugations, perfect Galois connections of TYPE OI between left and right semimodules (Section 2.3.2).

Although dioids carry us almost to our goal, working in complete idempotent semifields is necessary for the conjugations. This also has the beneficial side effect of providing algebraically-closed formulas for the adjoint-maps in terms of the completed idempotent semifields and their order duals (Section 2.3.3). We use Moreau's notation throughout in order to prove that it simplifies things considerably.

\subsubsection{Constructing Galois connections between idempotent semimodules}

The following crucial construction, due to Cohen et al. [6], is reminiscent of a similar one for binary residuated maps [14, §3.1.3]: Given a complete dioid $\mathcal{D}$, we call predual pair a complete left $\mathcal{D}$-semimodule $\mathcal{Y}$ together with a complete right $\mathcal{D}$-semimodule $\mathcal{X}$ equipped with a bracket to a complete $\mathcal{D}$-bisemimodule $\mathcal{Z},\langle\cdot \mid \cdot\rangle: Y \times X \rightarrow Z$, such that, for all $y \in Y, x \in X$ the maps $L_{y}: X \rightarrow$ $Z, x \mapsto\langle y \mid x\rangle$ and $R_{x}: Y \rightarrow Z, y \mapsto\langle y \mid x\rangle$ are, respectively, left and right linear, and residuated (see 2.1.1). 
For an arbitrary element $\varphi \in Z$, called the pivot, consider the residual maps ${ }_{\varphi}^{+} x=\left(\mathrm{R}_{x}^{\#}\right)(\varphi)$ and $y_{\varphi}^{+}=\left(\mathrm{L}_{y}^{\#}\right)(\varphi)$ 凹

$$
\begin{array}{ll}
\stackrel{+}{\varphi}^{+}: Y \rightarrow X & { }_{\varphi}^{+}: X \rightarrow Y \\
y_{\varphi}^{+}=\bigvee\{x \in X \mid\langle y \mid x\rangle \leq \varphi\} & { }_{\varphi}^{+} x=\bigvee\{y \in Y \mid\langle y \mid x\rangle \leq \varphi\}
\end{array}
$$

Proposition 2.8. $\left[{ }_{\varphi}^{+},{ }_{\varphi}^{+} \cdot\right]: \mathcal{Y} \leftrightharpoons \mathcal{X}$ is a Galois connection of TYPE OI.

Proof. From the properties of residuals $\langle y \mid x\rangle \leq \varphi \Longleftrightarrow x \leq y_{\varphi}^{+} \Longleftrightarrow y \leq{ }_{\varphi}^{+} x$ 6, proof of prop. 24], hence a Galois connection TYPE or by Definition 3

Recall that table 2 top right, summarizes the properties of such pairs of maps - their compositions are closures, they behave as pseudo-inverses of each other, both are antitone and join-inverting, etc. - and figure1 top right, depicts the morphisms and structures induced by them-the closure functions, the sets of closed elements $\bar{X}$ and $\bar{Y}$ and the perfect Galois connection between them.

Notice that the construction above is affected crucially by the choice of a suitable pivot $\varphi$ : if we consider the bracket to reflect a degree of relatedness between the elements of each pair, only those pairs $(y, x) \in Y \times X$ are considered by the connection whose degree amounts at most to $\varphi$. Therefore we can think of the pivot as a threshold for the pairs, or maximum allowed degree of existence.

We want the maps of equation (17) to fulfil the role of polars in FCA, hence in the following sections we will try to give algebraically closed forms for them.

\subsubsection{Reflexive semirings and conjugations over idempotent semimodules}

In equation (17), $\mathcal{X}$ and $\mathcal{Y}$ are both already complete lattices as well as free vector spaces. However, the closure lattices $\langle\bar{Y}, \leq\rangle={ }_{\varphi}^{+}(\mathcal{X})$ and $\langle\bar{X}, \leq\rangle=(\mathcal{Y})_{\varphi}^{+}$ do not generally agree with their ambient vector spaces in their joins, but only in their meets. In order to tighten the connection we introduce the following notion:

Definition 3. A left (resp. right) reflexive dioid, $(\mathcal{D}, \varphi)$, is a complete dioid with a bracket $\langle\cdot \mid \cdot\rangle: D \times D \rightarrow D$ with $\langle\lambda \mid \mu\rangle=\lambda \mu$ such that $\forall \lambda \in D$, ${ }_{\varphi}^{+}\left(\lambda_{\varphi}^{+}\right)=\lambda$ (resp. $\left({ }_{\varphi}^{+} \lambda\right)_{\varphi}^{+}=\lambda$ ). If such a dioid is both left and right reflexive we simply say that it is reflexive.

Cohen et al. 6, §4.3] prove that idempotent semifields are reflexive, and suggest that for the Boolean semiring we must choose $\varphi=0_{\mathcal{B}}$, the bottom in the order. For other idempotent semifields any invertible element may be chosen, for instance the multiplicative unit $\varphi=e_{\mathcal{D}}$. Notice that $\varphi$ need not be unique: if $(\mathcal{D}, \varphi)$ is reflexive, for any $\lambda \in D$ invertible, $(\mathcal{D}, \varphi \lambda)$ is reflexive.

\footnotetext{
${ }^{4}$ Notation $y_{\varphi}^{+},{ }_{\varphi}^{+} x$ emphasizes $\varphi$ as a parameter, whereas $\mathrm{R}_{x}^{\#}(\varphi), \mathrm{L}_{y}^{\#}(\varphi)$ emphasizes $x, y$ as parameters.
} 
Definition 4. (Conjugations) For $(\mathcal{D}, \varphi)$ a reflexive, complete, idempotent semiring, $\mathcal{Y}$ and $\mathcal{X}$ left and right semimodules over $\mathcal{D}$ and canonical bracket $\langle\cdot \mid \cdot\rangle_{\text {OI }}: Y \times X \rightarrow D,\langle y \mid x\rangle_{\text {OI }}=y \otimes x$ we define a conjugation to be the Galois connection of TYPE OI obtained from the maps in equation (17) for $\varphi=e_{\mathcal{K}^{\mathrm{d}}}$, and we write simply: $\left({ }^{\circledast},{ }^{\circledast} \cdot\right): \mathcal{Y} \sqcup \mathcal{X}$. For any other invertible element $\varphi$ we have the $\varphi$-conjugations: $\left(\cdot \stackrel{\circledast}{\varphi}, \frac{\circledast}{\varphi} \cdot\right): \mathcal{Y} \sqcup \mathcal{X}$.

For reflexive dioids the closure sets $\bar{X}$ and $\bar{Y}$ are actually (join-)subsemimodules of the corresponding spaces [6, propo. 28], hence conjugations and $\varphi$-conjugations for invertible $\varphi$ are perfect Galois connections .

Example 10. For $\overline{\mathbb{R}}_{\text {max },+}$ and $\overline{\mathbb{R}}_{\text {min },+}$ we have $y^{\circledast}:=-y$ and ${ }^{\circledast} x:=-x$.

Example 11. (Conjugations in matrix semimodules) For $(\overline{\mathcal{K}}, \varphi)$ a reflexive, complete, idempotent semifield, the idempotent semiring $\left(\mathcal{M}_{n}(\overline{\mathcal{K}}), I_{n}^{\mathrm{d}}\right)$ is reflexivewith $\left(I_{n}^{\mathrm{d}}\right)_{i j}=e$ if $i=j$, and $\top_{\overline{\mathcal{K}}}$ otherwise - hence the conjugations of Definition 团 exist for $R \in K^{g \times m}$ taken as either belonging to a left or right semimodule:

$$
R^{\circledast}=R \backslash I_{g}^{\mathrm{d}} \quad{ }^{\circledast} R=I_{m}^{\mathrm{d}} / R
$$

We also define the transpose $y^{\mathrm{T}}=\left(y^{-1}\right)^{\circledast}=\left(y^{\circledast}\right)^{-1}$ where $\cdot^{-1}: Y \rightarrow Y$ stands for inversion in the semimodule, and similarly for right semimodules .

The advantages of operating in such semimodules stem from:

Proposition 2.9. In semimodules over completed idempotent semifields $\left(\overline{\mathcal{K}}, \overline{\mathcal{K}}^{\mathrm{d}}\right)$ the following De Morgan-like laws hold for left (resp. right) conjugates:

$$
\begin{array}{ll}
(a \oplus b)^{\circledast}=a^{\circledast} \dot{\oplus} b^{\circledast} & (a \dot{\oplus} b)^{\circledast}=a^{\circledast} \oplus b^{\circledast} \\
(a \otimes b)^{\circledast}=b^{\circledast} \dot{\otimes} a^{\circledast} & (a \dot{\otimes} b)^{\circledast}=b^{\circledast} \otimes a^{\circledast}
\end{array}
$$

Proposition 2.10. In semimodules defined over completed idempotent semifields the following residuation laws hold:

$$
\begin{aligned}
& a \backslash c={ }^{\circledast} a \dot{\otimes} c={ }^{\circledast}\left(c^{\circledast} \otimes a\right) \\
& a \backslash c={ }^{\circledast} a \otimes c={ }^{\circledast}\left(c^{\circledast} \dot{\otimes} a\right) \\
& c / a=c \dot{\otimes} a^{\circledast}=\left(a \otimes{ }^{\circledast} c\right)^{\circledast} \\
& c / a=c \otimes a^{\circledast}=(a \dot{\otimes} \circledast c)^{\circledast}
\end{aligned}
$$

Hence operation in the opposite semirings $\overline{\mathcal{K}}^{\mathrm{op}},\left(\overline{\mathcal{K}}^{\mathrm{d}}\right)^{\mathrm{op}}$ is expressible in $\overline{\mathcal{K}}$, $\overline{\mathcal{K}}^{\mathrm{d}}$, and this extends to matrix semimodules: Therefore, by equation (17) we have $y^{\circledast}=y \backslash e_{\mathcal{K}},{ }^{\circledast} x=e_{\mathcal{K}} / x$, hence $y_{\varphi}^{\circledast}=y \backslash \varphi=y \backslash\left(e_{\mathcal{K}} \dot{\otimes} \varphi\right)=y^{\circledast} \dot{\otimes} \varphi$ and ${ }_{\varphi}^{\circledast} x=\varphi \dot{\otimes}{ }^{\circledast} x$. In practice, we ignore the side of the conjugation. 
Table 3: Brackets between left and right free semimodules defined over a complete idempotent semifield $\overline{\mathcal{K}}$ and its opposite $\overline{\mathcal{K}}^{\text {d }}$ with the aid of matrices defined over each semifield.

$\begin{array}{ll}\text { With range in } \overline{\mathcal{K}} & \text { With range in } \overline{\mathcal{K}}^{\mathrm{d}} \\ \langle\cdot|R| \cdot\rangle_{\mathrm{OI}}: \overline{\mathcal{K}}^{g} \times \overline{\mathcal{K}}^{m} \rightarrow \overline{\mathcal{K}} & {[\cdot|R| \cdot]_{\mathrm{OI}}:\left(\overline{\mathcal{K}}^{\mathrm{d}}\right)^{g} \times\left(\overline{\mathcal{K}}^{\mathrm{d}}\right)^{m} \rightarrow \overline{\mathcal{K}}^{\mathrm{d}}} \\ \langle y|R| x\rangle_{\mathrm{OI}}=y \otimes R \otimes x & {[y|R| x]_{\mathrm{OI}}=y \dot{\otimes} R \dot{\otimes} x} \\ \langle\cdot|R| \cdot\rangle_{\mathrm{OO}}: \overline{\mathcal{K}}^{g} \times\left(\overline{\mathcal{K}}^{\mathrm{d}}\right)^{m} \rightarrow \overline{\mathcal{K}} & {[\cdot|R| \cdot]_{\mathrm{OO}}:\left(\overline{\mathcal{K}}^{\mathrm{d}}\right)^{g} \times \overline{\mathcal{K}}^{m} \rightarrow \overline{\mathcal{K}}^{\mathrm{d}}} \\ \langle y|R| x\rangle_{\mathrm{OO}}=y \otimes R \otimes x^{-1} \cdot{ }^{-1} & {[y|R| x]_{\mathrm{OO}}=y \dot{\otimes} \dot{\otimes} x^{-1}} \\ \langle\cdot|R| \cdot\rangle_{\mathrm{IO}}:\left(\overline{\mathcal{K}}^{\mathrm{d}}\right)^{g} \times\left(\overline{\mathcal{K}}^{\mathrm{d}}\right)^{m} \rightarrow \overline{\mathcal{K}} & {[\cdot|R| \cdot]_{\mathrm{IO}}: \overline{\mathcal{K}}^{g} \times \overline{\mathcal{K}}^{m} \rightarrow \overline{\mathcal{K}}^{\mathrm{d}}} \\ \langle y|R| x\rangle_{\mathrm{IO}}=y^{-1} \otimes R \otimes x^{-1} & {[y|R| x]_{\mathrm{IO}}=y \dot{\theta}^{-1} \dot{\otimes} \dot{\otimes} x^{-1}} \\ \langle\cdot|R| \cdot\rangle_{\mathrm{II}}:\left(\overline{\mathcal{K}}^{\mathrm{d}}\right)^{g} \times \overline{\mathcal{K}}^{m} \rightarrow \overline{\mathcal{K}} & {[\cdot|R| \cdot]_{\mathrm{II}}: \overline{\mathcal{K}}^{g} \times\left(\overline{\mathcal{K}}^{\mathrm{d}}\right)^{m} \rightarrow \overline{\mathcal{K}}^{\mathrm{d}}} \\ \langle y|R| x\rangle_{\mathrm{II}}=y^{-1} \otimes R \otimes x & {[y|R| x]_{\mathrm{II}}=y^{-1} \dot{\otimes} R \dot{\otimes} x}\end{array}$

\subsubsection{Further Galois connections between idempotent subsemimodules}

The next challenge is to extend construction (17) to relations with values in $\overline{\mathcal{K}}$ and different modes of conceptualisation as suggested in section 2.1.3 From now on, consider a completed, idempotent semifield $\left(\overline{\mathcal{K}}, \overline{\mathcal{K}}^{\mathrm{d}}\right)$.

Proposition 2.11 (Dual pair induced by a matrix, Cohen et al. [6, §4.5]). Given a matrix $R \in \overline{\mathcal{K}}^{g \times m}$, the free complete right and left semimodules $\mathcal{X} \cong \overline{\mathcal{K}}^{m \times 1}$ and $\mathcal{Y} \cong \overline{\mathcal{K}}^{1 \times g}$ form a dual pair for the bracket $\langle y|R| x\rangle=y^{\mathrm{T}} \otimes R \otimes x$.

Corollary 2.12 (Galois connection induced by a matrix). For a specific $\varphi \in K$ define the maps

$$
y_{R, \varphi}^{+}=\bigvee\{x \mid\langle y|R| x\rangle \leq \varphi\} \quad{ }_{R, \varphi}^{+} x=\bigvee\{y \mid\langle y|R| x\rangle \leq \varphi\}
$$

then $\left[\cdot_{R, \varphi}^{+},{ }_{R, \varphi}^{+} \cdot\right]: \overline{\mathcal{K}}^{1 \times g} \sqcup \overline{\mathcal{K}}^{m \times 1}$ is a Galois connection of TYPE OI.

Proof. This follows from Propositions 2.8 and 2.11

Considering semimodule vectors and relations to take values in either $\overline{\mathcal{K}}$ or $\overline{\mathcal{K}}^{\mathrm{d}}$, and the brackets in Table 3 we claim,

Proposition 2.13. For $(\mathcal{K}, \varphi)$ a reflexive, idempotent semifield, 
1. for given $R \in \mathcal{M}_{g \times m}(\overline{\mathcal{K}})$, the brackets in the left column generate all possible types of Galois connections between the appropriate $\mathcal{Y}$ and $\mathcal{X}$ by composition with adequate conjugations.

2. for given $R \in \mathcal{M}_{g \times m}\left(\overline{\mathcal{K}}^{d}\right)$, the brackets in the right column generate all possible connections between the appropriate $\mathcal{Y}$ and $\mathcal{X}$ by composition with adequate conjugations.

Proof. For 1), bracket $\langle\cdot|R| \cdot\rangle_{\text {OI }}$ generates the conjugation of Proposition 2.12 above, which is a Galois connection of TYPE OI. Since $\langle\cdot|R| \cdot\rangle_{\text {II }}$ generates another TYPE OI between $\left(\overline{\mathcal{K}}^{\mathrm{d}}\right)^{g}$ and $\overline{\mathcal{K}}^{m}$, pre-composing with an inversion between $\overline{\mathcal{K}}^{g}$ and $\left(\overline{\mathcal{K}}^{\mathrm{d}}\right)^{g}$ as defined previously obtains a right adjunction, TYPE II, between $\overline{\mathcal{K}}^{g}$ and $\overline{\mathcal{K}}^{m}$. The procedure is exactly the same for TYPE OO and TYPE IO connections, using adequate compositions with conjugations on the left and right. For 2) the procedure is exactly the same starting from $[\cdot|R| \cdot]_{\mathrm{OI}}$, the bracket generating the Galois connection proper between $\left(\overline{\mathcal{K}}^{\mathrm{d}}\right)^{g}$ and $\left(\overline{\mathcal{K}}^{\mathrm{d}}\right)^{m}$.

\subsection{Generalising Formal Concept Analysis with idempotent semimodules}

To build a semiring-valued analogue of Formal Concept Analysis we need first a Galois connection proper, like the one provided by the construction in (17). However, the way polars work for this connection is not the way the polars of the original connection behave. This motivates creating $\overline{\mathcal{K}}$-Formal Concept Analysis as described below.

\subsection{1. $\overline{\mathcal{K}}$-Formal Concept Analysis}

Consider two indices $I$ and $J$ into two sets of objects $G=\left\{g_{i}\right\}_{i \in I}$ and attributes $M=\left\{m_{j}\right\}_{j \in J}$, respectively. We model ( $\overline{\mathcal{K}}$-valued $)$ sets of objects with vectors in a left $\overline{\mathcal{K}}$-semimodule, $y \in \mathcal{Y} \cong \overline{\mathcal{K}}^{I}$, and sets of attributes with vectors in a right $\overline{\mathcal{K}}$-semimodule, $x \in \mathcal{X} \cong \overline{\mathcal{K}}^{J}$, as generalisations of characteristic functions in the power sets $\mathbf{2}^{G}, \mathbf{2}^{M}$, respectively. For countable $|G|=g$ and $|M|=m$, these are simply row and column vectors.

Definition 5. Given a $\overline{\mathcal{K}}$-valued incidence between $G$ and $M, R \in \mathcal{K}^{I \times J}$, where $R(i, j)=\lambda$ reads as "object $g_{i}$ is incident with attribute $m_{j}$ in degree $\lambda$ " and dually "attribute $m_{j}$ is manifested in object $g_{i}$ to degree $\lambda$ ", the triple $(G, M, R)_{\overline{\mathcal{K}}}$ is called a $\overline{\mathcal{K}}$-valued formal context.

Consider the bracket $\langle x|R| y\rangle=y \otimes R^{-1} \otimes x$. The dually adjoint maps

$$
(y)_{R, \varphi}^{+}=\bigvee\{x \in X \mid\langle x|R| y\rangle \leq \varphi\} \quad \stackrel{+}{R, \varphi}^{+}(x)=\bigvee\{y \in Y \mid\langle x|R| y\rangle \leq \varphi\}
$$


are the polars of the Galois connection $\left[(\cdot)_{R, \varphi}^{+},{ }_{R, \varphi}^{+}(\cdot)\right]: \mathcal{Y} \sqcup \mathcal{X}$. In completed idempotent semifields we can give them closed forms:

$$
\begin{aligned}
(y)_{R, \varphi}^{+} & =\left(y \otimes R^{-1}\right) \backslash \varphi & { }_{R, \varphi}^{+}(x) & =\varphi /\left(R^{-1} \otimes x\right) \\
& =R^{\mathrm{T}} \dot{\otimes} y^{\circledast} \dot{\otimes} \varphi & & =\varphi \otimes x^{\circledast} \otimes R^{\mathrm{T}}
\end{aligned}
$$

Definition 6. A formal $\varphi$-concept of the formal context $(G, M, R)_{\overline{\mathcal{K}}}$ is a pair $(a, b)_{\varphi} \in Y \times X$ such that $(a)_{R, \varphi}^{+}=b$ and ${ }_{R, \varphi}^{+}(b)=a$, where $a$ is the $\varphi$-extent and $b$ the $\varphi$-intent. We call $\mathfrak{B}^{\varphi}(G, M, R)_{\overline{\mathcal{K}}}$ the set of all concepts and $\varphi$ its maximum threshold of existence.

If $\left(a_{1}, b_{1}\right)_{\varphi}\left(a_{2}, b_{2}\right)_{\varphi}$ are $\varphi$-concepts of a context, they are ordered by the relation $\left(a_{1}, b_{1}\right)_{\varphi} \leq\left(a_{2}, b_{2}\right)_{\varphi} \Longleftrightarrow a_{1} \preccurlyeq a_{2} \Longleftrightarrow b_{1} \preccurlyeq^{\mathrm{d}} b_{2}$, called the hierarchical order. Then:

Theorem 2.14. [Basic theorem of $\overline{\mathcal{K}}$-valued Formal Concept Analysis, 35$]$ The set of concepts of a $\overline{\mathcal{K}}$-valued formal context $(G, M, R)_{\overline{\mathcal{K}}}$ with the hierarchical order is a complete lattice $\underline{\mathfrak{B}}^{\varphi}(G, M, R)_{\overline{\mathcal{K}}}$ with infima and suprema given by

$$
\begin{aligned}
& \bigwedge_{t \in T}\left(a_{t}, b_{t}\right)_{\varphi}=\left(\sum_{t \in T}^{\bullet} a_{t},\left[_{R, \varphi}^{+}\left[\sum_{t \in T} \cdot b_{t}\right]\right]_{R, \varphi}^{+}\right) \\
& \bigvee_{t \in T}\left(a_{t}, b_{t}\right)_{\varphi}=\left(\sum_{R, \varphi}^{+}\left[\left[\sum_{t \in T} a_{t}\right]_{R, \varphi}^{+}\right], \sum_{t \in T}^{\bullet} b_{t}\right)
\end{aligned}
$$

The next corollary relates $\mathcal{K}$-FCA and standard FCA:

Corollary 2.15. FCA is $\mathbb{B}-F C A$. Formal contexts and formal concepts are 0contexts and 0 -concepts.

Proof. Use $\overline{\mathcal{K}}:=\mathbb{B}$ in the constructions above, when $R=I$ is boolean. As requested in Section 2.3.1 for $\mathbb{B}$ we must choose $\varphi:=\epsilon_{\mathbb{B}}=\mathbf{0}$, hence

$$
a^{\prime}=(a)_{I, 0}^{+}=I^{\mathrm{T}} \dot{\otimes} a^{\circledast} \quad \quad b^{\prime}={ }_{I, 0}^{+}(b)=b^{\circledast} \dot{\otimes} I^{\mathrm{T}}
$$

so $\underline{\mathfrak{B}}(G, M, I)=\mathfrak{B}^{0}(G, M, I)_{\mathbb{B}}$.

\subsubsection{The structural lattice of a $\overline{\mathcal{K}}$-Concept Lattice}

Theorem 2.14 is an incomplete analogue of the basic theorem of FCA: the latter's universal representation capabilities are missing, yet this seems crucial for applications: $\underline{\mathfrak{B}}^{\varphi}(G, M, R)_{\overline{\mathcal{K}}}$ may be a huge lattice - potentially with infinite concepts. We would like to obtain approximation to these lattices with the same "structure". 
Given the polars in (22), the dually isomorphic closure lattices are

$$
\overline{\mathcal{Y}}=\left\{{ }_{R, \varphi}^{+} x=\varphi \dot{\otimes} x^{\circledast} \dot{\otimes} R^{\mathrm{T}} \mid x \in X\right\} \quad \overline{\mathcal{X}}=\left\{y_{R, \varphi}^{+}=R^{\mathrm{T}} \dot{\otimes} y^{\circledast} \dot{\otimes} \varphi \mid y \in Y\right\}
$$

where $Y \cong K^{G}$ is the free space of object sets and $X \cong K^{M}$ is the free space of attribute sets. Let the singleton sets of objects (row vectors), $g_{i}=$ $\left[\perp \cdots e_{i} \cdots \perp\right]$, and attributes (column vectors), $m_{j}=\left[\perp \cdots e_{j} \cdots \perp\right]^{\mathrm{T}}-$ bases of their respective (free) spaces - be mapped through the polars:

$$
W_{\overline{\mathcal{Y}}}=\left\{u_{j}={ }_{R, \varphi}^{+}\left(m_{j}\right) \mid j \in J\right\} \quad W_{\overline{\mathcal{X}}}=\left\{v_{i}=\left(g_{i}\right)_{R, \varphi}^{+} \mid i \in I\right\}
$$

to obtain generator sets for the closure lattices:

$$
\overline{\mathcal{Y}}=\left\{\sum_{j \in J} \lambda_{j} \otimes u_{j} \mid \lambda_{j} \in K\right\} \quad \overline{\mathcal{X}}=\left\{\sum_{i \in I} \mu_{i} \otimes v_{i} \mid \mu_{i} \in K\right\}
$$

Since the $\vee$-irreducibles are part of each set of generators we may test the latter to find the former:

$$
\mathcal{J}(\overline{\mathcal{Y}}) \subseteq W_{\bar{Y}} \quad \mathcal{J}(\overline{\mathcal{X}}) \subseteq W_{\overline{\mathcal{X}}}
$$

Next recall that the Galois connection of (21) is $\vee$-inverting, therefore, the images of the $\vee$-irreducibles are $\wedge$-irreducibles of the dual lattice:

$$
\mathcal{M}(\overline{\mathcal{Y}})=_{R, \varphi}^{+}[\mathcal{J}(\overline{\mathcal{X}})] \quad \mathcal{M}(\overline{\mathcal{X}})=[\mathcal{J}(\overline{\mathcal{Y}})]_{R, \varphi}^{+}
$$

Alternatively we may think of the product of each pair of join- and meetirreducible sets as being comprised of $\vee$-irreducible (resp. $\wedge$-irreducible) concepts with definitions resembling those of the standard theory. Consider the mappings $\tilde{\gamma}_{R, \varphi}^{+}: Y \rightarrow \mathfrak{B}^{\varphi}(G, M, R)_{\overline{\mathcal{K}}}$ and $\tilde{\mu}_{R, \varphi}^{+}: X \rightarrow \mathfrak{B}^{\varphi}(G, M, R)_{\overline{\mathcal{K}}}$

$$
\left.\tilde{\gamma}_{R, \varphi}^{+}\left(g_{i}\right)=\left(\begin{array}{l}
+ \\
R, \varphi
\end{array}\left[g_{i}\right]_{R, \varphi}^{+}\right],\left[g_{i}\right]_{R, \varphi}^{+}\right) \quad \tilde{\mu}_{R, \varphi}^{+}\left(m_{j}\right)=\left({ }_{R, \varphi}^{+}\left[m_{j}\right],\left[\begin{array}{l}
+ \\
R, \varphi
\end{array}\left[m_{j}\right]\right]_{R, \varphi}^{+}\right)
$$

Therefore, the following lattices seem $\mathcal{K}$-FCA analogues of canonical lattices associated to a standard context:

Definition 7. [ $\varphi$-structural lattices, $\underline{34}]$ The $\varphi$-structural lattice $\underline{\mathfrak{B}}\left(G, M, I_{R, \varphi}^{+}\right)$of a $\overline{\mathcal{K}}$-Formal Context $(G, M, R)_{\overline{\mathcal{K}}}$ is the Concept Lattice of $\left(G, M, I_{R, \varphi}^{+}\right)$, where

$$
I_{R, \varphi}^{+}(i, j)=\tilde{\gamma}_{R, \varphi}^{+}\left(g_{i}\right) \leq \tilde{\mu}_{R, \varphi}^{+}\left(m_{j}\right)
$$

Hence, $\overline{\mathcal{K}}$-Formal Contexts are not represented by a single concept lattice, but a whole family as $\varphi$ ranges along the invertible elements in the semifield. 


\section{Application: the Exploratory Analysis of Confusion Matrices}

To illustrate the use of extended, generalised FCA we use the problem of analysing confusion matrices, already tackled in 35], being a summary of the performance of multiple input-pattern, multiple output-pattern classifiers. This was already attempted by Düntsch and Gediga [10] but we feel it fell short of providing a generalisable scheme for interpreting relations.

Consider a classifier accepting patterns labelled with a set of stimuli $G$ and returning one label from a set of responses $M$. A confusion matrix - or contingency table - is a matrix $C M \in \mathbb{N}_{0}$ describing how many times the experiment of classifying a pattern labelled $g_{i}$ returned label $m_{j}$.

We will introduce the analysis confusion matrices in two steps: first we present an extended analysis of binarised confusion matrices and then an extended analysis of $\overline{\mathcal{K}}$-contexts.

\subsection{Extended Formal Concept Analysis of binary confusion matrices}

For a small example, consider: $G=\{$ Dog, Cat, Rab(bit) $\}$, where the uppercase letters emphasise that these are stimulus labels, and $M=\{$ dog?, cat?, rab? where the emphasis is on the uncertainty of the response label. The left of Figure [3illustrates one such matrix with the usual hypothesis $g=m$.

In this section we will consider a trivial binarisation of this counting scheme where $\left(g_{i}, m_{j}\right)$ is set to 1 if that event ever happened more than twice. The four broad-sense formal contexts related to $I^{C M}$ are illustrated in Figure 4 and the broad-sense concept lattices in Figure 5

For each particular application we need to know how to interpret broadsense formal concepts in the context of the particular relation being analysed, and how to glean overall information from the broad-sense concept lattices.

\subsubsection{The interpretation of broad-sense concepts}

Let $C \in 2^{G \times M}$ be a binary confusion or contingency matrix, between stimuli and responses with $\mathrm{gCm}$ being interpreted as stimulus $\mathrm{g}$ evokes response $\mathrm{m}$ or response $m$ is evoked by stimulus $g$. For sets of stimuli $y \in 2^{G}$ and responses $x \in 2^{M}$, the Galois connections and the interpretation of the (dual) adjoint functions induced by the confusion matrix are:

1. TYPE OO. A Galois adjunction on the right $\left[(\cdot)_{C}^{\exists},{ }_{C}^{\forall}(\cdot)\right]: G \leftrightharpoons M$ where the adjoint maps of the object neighbourhood lattice can be read as:

$$
\begin{aligned}
& m \in Y_{C}^{\exists} \Longleftrightarrow \text { some stimulus } g \text { in } Y \text { evokes response } m \\
& g \in{ }_{C}^{\forall} X \Longleftrightarrow \text { each response } m \text { evoked by stimulus } g \text { is in } X
\end{aligned}
$$

2. TYPE OI. A Galois connection proper $\left[(\cdot)_{C}^{+},{ }_{C}^{+}(\cdot)\right]: G \sqcup M$ where the dually adjoint maps of FCA can be read as:

$m \in Y_{C}^{+} \Longleftrightarrow$ response $m$ is evoked by every stimulus $g \in Y$

$g \in{ }_{C}^{+} X \Longleftrightarrow$ stimulus $g$ evokes every response $m$ in $X$ 
3. TYPE IO. A co-Galois connection $\left[(\cdot)_{C}^{-}, \bar{C}_{C}^{-}(\cdot)\right]: G \rightarrow M$ where the dually adjoint maps can be read as:

$$
\begin{aligned}
m \in Y_{C}^{-} & \Longleftrightarrow \text { not all stimuli in } Y^{\mathbf{c}} \text { evoke response } m \\
& \Longleftrightarrow \text { response } m \text { does not evoke every stimulus } g \text { in } Y^{\mathbf{c}} \\
g \in{ }_{C} X & \Longleftrightarrow \text { not all responses in } X^{\mathbf{c}} \text { are evoked by stimulus } g \\
& \Longleftrightarrow \text { stimulus } g \text { does not evoke every response } m \text { in } X^{\mathbf{c}}
\end{aligned}
$$

4. TYPE IO. A Galois adjunction on the left $\left[(\cdot)_{C}^{\forall},{ }_{C}^{\exists}(\cdot)\right]: G \rightleftharpoons M$ where the adjoint maps of an attribute neighbourhood lattice can be read as:

$$
\begin{aligned}
& m \in Y_{C}^{\forall} \Longleftrightarrow \text { each stimulus } g \text { evoking response } m \text { is in } Y \\
& g \in{ }_{C}^{\exists} X \Longleftrightarrow \text { some response } m \text { in } X \text { is evoked by stimulus } g
\end{aligned}
$$

\subsubsection{The interpretation of the broad-sense concept lattices}

The standard formal context for $C$ is that of Subfigure $4 b$ and its concept lattice is illustrated in Figure 5b. For instance (Rab,rab?) attests that only "Rab(bits)" evoke "rab?(bits)", and only "rab?" are evoked by "Rab". This is a rather special kind of concept, collecting stimuli "confused" with just one response, that is, essentially not confused with anything else. More generally:

- We look upwards from single stimulus (object) concepts and find those responses (attributes) evoked by it. For instance, $(\{D o g\},\{d o g$ ?, cat? $\})$ says that "Dog" evokes "dog?" and "cat?", and the only thing that can evoke both "dog?" and "cat?" is a "Dog".

- We look downwards from single response (attribute) concepts to find which input patterns (objects) are confused with it. For instance, $(\{D o g, C a t\}$, $\{$ cat?\}) states that "Dog" and "Cat" can be invoked by "cat?".

With this in mind, recall from Corollary 2.5 that the (broad-sense) concept lattices arising from the different Galois connections can be obtained as the concept lattices of contexts systematically derived from $(G, M, C)$. We use some non-standard notation to represent these contexts and lattices:

- When an object or attribute is written with an overline the formal context actually is predicating the incidence of the complement of that singleton, that is $\{\overline{R a b}\}=\{$ Dog, Cat $\},\{\overline{c a t} ?\}=\{d o g ?, r a b ?\}$.

- A set of overlined objects or attributes denotes the intersection of the unitary complements: $\left\{\overline{g_{1}}, \ldots \overline{g_{n}}\right\}=\overline{g_{1}} \cap \ldots \overline{g_{n}}$. In practice this entails the change from a base of singletons to a base of complements of singletons.

- Reading filters and ideals off the diagram should be done with the adequate preservation and/or inversion of joins and meets following Proposition 2.1. 
Consider the case of the object neighbourhood lattice $\underline{\mathfrak{N}}^{G}(G, M, C) \cong$ $\underline{\mathfrak{B}}_{\mathrm{OO}}(G, M, C)$, shown in Figure $5 \mathbf{b}$ This is the (standard) concept lattice of the context illustrated in Figure 4a $\mathfrak{B}\left(G, M^{-}, \phi\right)$, where we use normal objects and complemented attribute sets. In general,

- for stimulus (object) concepts we read off the intent those responses never evoked by that stimulus: ( $\{C a t\},\{\overline{d o g}$ ? $\overline{r a b}$ ? $\}$ ), states that "Cat" does not evoke either "dog?" or "rab?", and anything that does not evoke either "dog?" or "rab?" can only be "Cat".

- for complemented response (attribute) concepts, we read off the extent those stimuli that never evoke that response: in $(\{D o g, C a t\},\{\overline{r a b} ?\})$, "Dog" and "Cat" never evoke "rab?", which is the only thing not evoked by either.

The case of attribute neighbourhood lattices $\underline{\mathfrak{N}}^{M}(G, M, C) \cong \underline{\mathfrak{B}}_{\text {II }}(G, M, C)$ of Figure $5 \mathrm{~d}$ is a mirror image of the previous one above. This is the concept lattice $\mathfrak{B}\left(G^{-}, M, C\right)$ in Figure 4d] with complemented object sets and usual attributes. In general:

- for complemented stimulus (object) concepts, we read off the intent those responses never evoked by that stimulus: $(\{\overline{\mathrm{Cat}}\},\{d o g$ ?, rab?\}), those things not evoked by a "Cat" are "dog?" and "rab?", and whatever these two are evoked by cannot be a "Cat".

- for response (attribute) concepts, we read off the extent those stimuli that never evoke the response: $(\{\overline{D o g}, \overline{C a t}\},\{r a b ?\})$, says that "rab?" is not evoked by "Dog" and "Cat" which are the only things not evoking it.

Finally we find the unrelatedness lattice $\underline{\mathfrak{B}}^{U}(G, M, C)=\underline{\mathfrak{B}}_{\mathrm{IO}}(G, M, C)$ in Figure $5 \mathfrak{c}$ which is the concept lattice $\underline{\mathfrak{B}}\left(G^{-}, M^{-}, C\right)$ considering both complemented object and attribute sets.

Another way of looking into things is to consider particular concepts, for instance consider a single "Cat":

- from TYPE OI concepts, we know it evokes "cat?", but the latter can also be evoked by "Dog".

- from the TYPE OO lattice, we know it can never evoke "dog?" or "rab?", the only tag of this kind.

- from TYPE II concepts, we know that something evoked as "rab?" and "dog?" cannot be a "Cat", and finally

- from TYPE IO concepts, nothing can be found for "Cat". 


\subsection{Extended analysis of structural lattices from perceptual confusion matrices}

For a non-binary confusion matrix we cannot carry the extended analysis as before, but we can first obtain a motivated binarisation and then try to analyse it extendedly. The procedure has three conceptual steps: first casting the countbased matrix into a $\overline{\mathcal{K}}$ matrix; then exploring the lattice in the $\varphi$ parameter to obtain a (binary) structural lattice; and finally performing an extended Formal Concept Analysis as done in the previous section. We will carry it on perceptual confusion matrices from Miller and Nicely [25].

The first step is akin to a data conditioning procedure. In the case of confusion matrices, selecting to explore the mutual information provided by the count matrix can be motivated independently [25] $C M\left(g_{i}, m_{j}\right)=\log \frac{\hat{P}_{G M}\left(g_{i}, m_{j}\right)}{\hat{P}_{G}\left(g_{i}\right) \hat{P}_{M}\left(m_{j}\right)}$ where $\hat{P}_{G M}, \hat{P}_{G}$ and $\hat{P}_{M}$ are, for instances, maximum-likelihood estimates of the corrected joint-probabilities stemming from the count matrix. This embeds the entries the new $C M$ into $\overline{\mathbb{R}}_{\max ,+}$ and we will only be concerned in the rest of this section with the other two steps.

\subsubsection{Lattice exploration procedure}

In $\left(\overline{\mathbb{R}}_{\max ,+}\right)$-Formal Concept Analysis, the procedure that selects the formal concepts depends on the threshold $\varphi$. Therefore, typically the calculation of the structural matrix in Definition 17 must be carried out a number of times, one for each choice of $\varphi$ that is deemed interesting, a process we call lattice exploration.

Since the complexity of a concept lattice can be roughly approximated by the cardinality of the concept set, or concept count, Figure 6] shows the evolution of the number of concepts of $I_{C M, \varphi}^{+}$(dual adjoints, dotted lines) and $干_{C M, \varphi}^{+}$(adjoints, solid line) with $\varphi$, where the top graph presents the experiment with good quality speech (Signal-to-Noise Ratio of $12 \mathrm{~dB}$ ) and the bottom one, another with poor quality (Signal-to-Noise Ratio of -12dB) .

For the dual adjoints, as we decrease the threshold of existence, $\varphi$, more confusions are brought into the picture producing complex structural lattices with a higher number of nodes. Eventually, a peak is reached where the consideration of lower thresholds does not increase the number of concepts. If, on the other hand, a larger value of $\varphi$ is chosen, the number of concepts will be reduced offering a much simpler structural lattice showing the most prominent confusions.

The two adjunctions (solid lines) however, exhibit the opposite trend, producing more complex lattices as the threshold $\varphi$ increases. Therefore, we find that starting from the smallest $\varphi$ and increasing its values, progressively brings into the picture more negative confusions.

The evolution of the number of concepts as explained above suggests a method for exploring structural lattices:

1. First, begin by observing the most salient properties of the system, that is, those lattices obtained with higher values of the threshold $\varphi$ for the connections and lower values for the adjunction. 
2. Subsequently, try to bring more detail into the picture by decreasing (resp. increasing) the value of the threshold for connections (resp. adjunctions) so as to progressively increase the number of concepts.

3. Choose representative values of $\varphi$ for Galois connections and adjunctions so that lattices of $I_{C M, \varphi}^{+}$and ${{ }_{C}}_{C M, \varphi}^{+}$have approximately the same number of concepts.

Figure 7 provides three types of structural lattices (as TYPE IO is highly redundant if the other three are present) for the confusion matrix of table VI (25]) introduced in the previous subsection.

\subsubsection{Extended analysis of confusion patterns}

To begin with, the TYPE OI Galois connection lattice in the middle of the figure shows several groups of consonantal groups independent of each other in terms of confusions. For example, nasal sounds $(/ m /$ and $/ n /)$ on the right, the unvoiced plosives $(/ p /, / t /, / k /)$ and unvoiced fricatives $(/ f /$ and $/ t h /, / s /$ and $/ s h /)$ are clearly separated from the rest of the voiced sounds on the left.

The left adjunction lattice at the top provides information of the stimuli which evoke the least number of erroneous responses. In particular, neither $/ m /$ nor $/ n /$, situated very low in the lattice evoke responses like $/ \mathrm{sh} / ?, / p /$, $/ k /$ ? or $/ f /$ ? (at this given $\varphi$ ). Additionally, $/ m /$ never evokes $/ z /$ ? and $/ n /$ never evokes $/ t h /$ ? or $/ t / ?$.

At the bottom of the figure, the right adjunction lattice provides information of which responses are the least evoked and their stimuli. The most salient characteristic observed here is that $/ p / ?, / k / ?$ and $/ f / ?$ are the responses evoked by the least stimuli as they appear near the top element.

\section{Results and Discussion}

In this paper we try to give new answers to the questions of extending the types of concepts considered in Formal Concept Analysis and generalising the type of incidences allowed in the lattice construction process. In particular we have provided:

- Extensions of Formal Concept Analysis (FCA) to deal with all combinations of closure and kernel operators generating broad-sense concept lattices.

- Methods to build such extended concept lattices for the boolean case reduced to the use of techniques developed for standard FCA.

- A basic formulation for extending also the generalised case where the incidence takes values in an idempotent semifield, $\overline{\mathcal{K}}$-Formal Concept Analysis.

- examples of how to use these techniques in the quantitative analysis of binary and semiring-valued phonetic confusion matrices, . 
The use of alternative Galois connections to extend Formal Concept Analysis was inspired by the work of Düntsch and Gediga [10, 11], Erné [12] and Erné et al. [13]. Our notation for the adjoints of Galois connections is heavily influenced by the latter, which seems to be well-known and cited. We have only meddled in here to give a more mnemonic notation emphasising the composition with dual order isomorphisms.

Using the polar for the neighbourhood of objects is not new in the FCA community, but ours is the first presentation of what we believe is the "correct" pairing of all maps induced by a binary relation, reducing the procedure for extended Formal Concept Analysis on a context to standard Formal Concept Analysis on contexts systematically derived from the original one.

We also provided algebraic formulae for the construction of Galois connections of all four different types - viz.. left and right adjunctions, Galois connections proper and co-Galois connections-between semimodules over reflexive idempotent semifields. The existence of these Galois connections allowed us to generalise and extend standard Formal Concept Analysis over binary incidences to extended $\overline{\mathcal{K}}$-Formal Concept Analysis over incidences with values in a complete idempotent semifield $\mathcal{K}$. Although such semifields turn out to be incomplete, we imported a construction to complete them, along with a convenient notation for expressing all Galois connection operators in matrix algebra. In the case of max-plus algebra, our procedure suggests that the proper way of operating with these Galois connections is to use max-min-plus algebra $\overline{\mathbb{R}}_{\max , \min ,+}$ that subsumes the completed idempotent semifields $\overline{\mathbb{R}}_{\max ,+}$ and $\overline{\mathbb{R}}_{\text {min.+. }}$.

A notation reminiscent of Moreau's was already used by Singer [29, 30, 31] in relation to co-Galois connections (TYPE IO) and the completions of certain idempotent semigroups, but was not explored systematically therein, and only cursory mention of the isomorphisms being monotone or antitone is done. An alternative notation for completed idempotent semirings - not just $\overline{\mathbb{R}}_{\max ,+}$-was proposed by Cuninghame-Green [7] and later taken and developed by several others, notably by [28, 21], but we believe Moreau's notation is more symmetric, besides having the precedence, at least for $\overline{\mathbb{R}}_{\max ,+}$ and $\overline{\mathbb{R}}_{\min ,+}$. The notation we use here mixes well with matrix calculations, inversions and conjugations.

$\overline{\mathcal{K}}$-Formal Concept Analysis stems from the work of Cohen et al. []], but it has a precedent - much later noticed by us - in the work of Cuninghame-Green 7], although it was not developed therein as an data exploration technique. The main scheme of extending FCA by combining a basic Galois connection proper plus the Cuninghame-Green conjugation for obtaining different types of Galois connections is already looming in [6, 16], but, to the extent of our knowledge, this paper is the first journal presentation of these techniques for data mining. Of course, such is exactly the way the right-axiality and the co-Galois connection were introduced in 13], but only for subsets of $2^{G}$ and $2^{M}$, whose generalisation to other semirings is not straightforward.

Düntsch and Gediga [10, 11] inspired the use of different conceptualization modes for the analysis of confusion matrices, although their work actually deals with dissimilarity matrices. However, their consideration of "confusions" does not involve the pictorial representations of the lattices here described, nor the 
consideration of degrees of incidence.

Since Fuzzy- and $\mathcal{K}$-Formal Concept Analysis seem to stem from double residuated lattices, a brief note comparing both kinds of generalisation is in order. Note that the theoretical basis of both Fuzzy- and $\mathcal{K}$-Formal Concept Analysis has been already extended beyond that of biresiduated lattices by Abdel-Hamid and Morsi 1]. However, the concrete constructions that result in standard, Fuzzy- and $\overline{\mathcal{K}}$-Formal Concept Analysis are still missing in this new framework.

Fuzzy Formal Concept Analysis is not a unique technique, but rather a family of them [see 22, 24, for reviews of their relationships], and it has lately been augmented to consider multiple domains (lattices) or non-commutatively generated polars, among other generalisations 24, 23]. But to our knowledge, the fuzzy-Formal Concept Analysis community has not yet used the alternate modes of conceptualisation we propose here - note that the connections of multiadjoint concept lattices seem all to be of TYPE OI.

As stated in Section 2.2.1 "fuzzy semirings" are instances of doubly idempotent semirings, which are as far apart from idempotent semifields as idempotent semirings can be [19]. Idempotent semifields all have a unit element for multiplication different to either top or bottom, whereas fuzzy semirings have an idempotent multiplication: we believe this will prove a key aspect in choosing either type of semiring for different applications.

\section{Conclusions}

In this paper we set out to extend conceptualisation modes for standard and generalised Formal Concept Analysis.

For binary incidences, the consideration of alternate forms of conceptualisation, called extended Formal Concept Analysis, can be effectively done with techniques based in standard FCA and adequate, alternative interpretations of formal concepts.

For incidences with values in a complete semifield $\overline{\mathcal{K}}$ a similar construction exists, $\overline{\mathcal{K}}$-Formal Concept Analysis, but its effectiveness is challenged by the existence of a free parameter $\varphi$ acting as a threshold on the existence of concepts.

An effective way to extend $\overline{\mathcal{K}}$-FCA is to explore on $\varphi$ and carry out extended FCA on the structural lattice for a representative $\varphi$.

\section{Acknowledgements}

This work has been partially supported by the Spanish Government-Comisión Interministerial de Ciencia y Tecnología project 2008-06382/TEC and 200802473/TEC and the regional project (Comunidad Autónoma de Madrid - UC3M) CCG08-UC3M/TIC-4457.

We would like to thank M. Erné for providing initial input for Galois connection diagrams in section 2.1.1] We would also like to thank the reviewers of early versions of this paper. 


\section{References}

[1] Abdel-Hamid, A. A. and Morsi, N. N. (2003). Associatively tied implications. Fuzzy Sets and Systems, 136(3), 291-311.

[2] Becker, P. and Correia, J. (2005). The ToscanaJ suite for implementing conceptual information systems. In Formal Concept Analysis: Foundations and Applications, volume LNAI 2636, pages 325-348.

[3] Bělohlávek, R. (1999). Lattice generated by binary fuzzy relations. Tatra Mt. Mathematical Publications, 16, 11-19.

[4] Birkhoff, G. (1967). Lattice theory. American Mathematical Society, 3rd ed. edition.

[5] Burusco, A. and Fuentes-González, R. (1994). The study of the L-fuzzy Concept Lattice. Mathware and Soft Computing, 1(3), 209-218.

[6] Cohen, G., Gaubert, S., and Quadrat, J.-P. (2004). Duality and separation theorems in idempotent semimodules. Linear Algebra and Its Applications, 379, 395-422.

[7] Cuninghame-Green, R. (1979). Minimax Algebra. Number 166 in Lecture notes in Economics and Mathematical Systems. Springer.

[8] Davey, B. and Priestley, H. (2002). Introduction to lattices and order. Cambridge University Press, Cambridge, UK, 2nd edition.

[9] Denecke, K., Erné, M., and Wismath, S., editors (2004). Galois Connections and Applications. Number 565 in Mathematics and Its Applications. Kluwer Academic, Dordrecht, Boston and London.

[10] Düntsch and Gediga, G. (2002). Modal-style operators in qualitative data analysis. Technical Report CS-02-15, Department of Computer Science, Brock University, St. Catharines, Ontario, Canada.

[11] Düntsch, I. and Gediga, G. (2003). Approximation operators in qualitative data analysis. In H. de Swart et al., editor, Theory and Applications of Relational Structures as Knowledge Instruments, volume 2929 of Lecture Notes in Computer Science, pages 214-230. Springer.

[12] Erné, M. (2004). Adjunctions and Galois connections: Origins, History and Development, pages 1-138. In [9].

[13] Erné, M., Koslowski, J., Melton, A., and Strecker, G. (1993). A primer on Galois connections. In A. Todd, editor, Proceedings of the 1991 Summer Conference on General Topology and Applications in Honor of Mary Ellen Rudin and Her Work, volume 704 of Annals of the New York Academy of Sciences, pages 103-125, Madison, WI, USA. New York Academy of Science. 
[14] Galatos, N., Jipsen, P., Kowalski, T., and Ono, H. (2007). Residuated lattices: an algebraic glimpse at substructural logics, volume 151 of Studies in Logic and the Foundations of Mathematics. Elsevier.

[15] Ganter, B. and Wille, R. (1999). Formal Concept Analysis: Mathematical Foundations. Springer, Berlin, Heidelberg.

[16] Gaubert, S. (1998). Two lectures on max-plus algebra. Support de cours de la 26-iéme École de Printemps d'Informatique Théorique. http://amadeus.inria.fr/gaubert/papers.html.

[17] Golan, J. S. (1999a). Power Algebras over Semirings. With Applications in Mathematics and Computer Science, volume 488 of Mathematics and its applications. Kluwer Academic, Dordrecht, Boston, London.

[18] Golan, J. S. (1999b). Semirings and their Applications. Kluwer Academic.

[19] Gondran, M. and Minoux, M. (2008). Graphs, Dioids and Semirings. New Models and Algorithms. Operations Research Computer Science Interfaces series. Springer.

[20] Groh, B. and Wille, R. (2000). Lattices of triadic concept graphs. Conceptual Structures: Logical, Linguistic, and Computational Issues. Proceedings of ICCS 2000., LNAI 1867, 332-341.

[21] Heidergott, B., Olsder, G. J., and van der Woude, J. (2006). Max Plus at Work. Modeling and Analysis of Synchronized Systems: A Course on MaxPlus Algebra and Its Applications. Princeton series in Applied Mathematics. Princeton University Press.

[22] Krajci, S. (2005). A generalized concept lattice. Logic Journal of IGPL, $\mathbf{1 3}(5), 543$.

[23] Medina, J. and Ojeda-Aciego, M. (2010). Multi-adjoint t-concept lattices. Information Sciences, 180(5), 712-725.

[24] Medina, J., Ojeda-Aciego, M., and no, J. R.-C. (2009). Formal concept analysis via multi-adjoint concept lattices. Fuzzy Sets and Systems, 160(2), $130-144$.

[25] Miller, G. A. and Nicely, P. E. (1955). An analysis of perceptual confusions among some English consonants. Journal of the Acoustical Society of America, 27(2), 338-352.

[26] Moreau, J. J. (1970). Inf-convolution, sous-additivité, convexité des fonctions numériques. J. Math. pures et appl., 49, 109-154.

[27] Pollandt, S. (1997). Fuzzy-Begriffe. Formale Begriffsanalyse unscharfer Daten. Springer, Heidelberg. 
[28] Ritter, G., Sussner, P., and de Leon, J. D. (1998). Morphological associative memories. IEEE Transactions on Neural Networks, 9(2), 281-293.

[29] Singer, I. (1997). Abstract Convex Analysis. Monographs and Advanced Texts. Wiley-Interscience.

[30] Singer, I. (2003a). (*,s)-dualities. Journal of Mathematical Sciences, $\mathbf{1 1 5}(4), 2506-2541$.

[31] Singer, I. (2003b). Some relations between linear mappings and conjugations in idempotent analysis. Journal of Mathematical Sciences, 115(5), 2610-2630.

[32] Taylor, P. (1999). Practical Foundations of Mathematics. Number 59 in Cambridge studies in Advanced Mathematics. Cambridge University Press.

[33] Valverde-Albacete, F. J. and Peláez-Moreno, C. (2006). Towards a generalisation of Formal Concept Analysis for data mining purposes. In R. Missaoui and J. Schmid, editors, Concept Lattices. Proceedings of the International Conference on Formal Concept Analysis, ICFCA06, (Dresden, Germany), volume 3874 of $L N A I$, pages 161-176, Berlin, Heidelberg. Springer.

[34] Valverde-Albacete, F. J. and Peláez-Moreno, C. (2007a). Further Galois connections between semimodules over idempotent semirings. In J. Diatta and P. Eklund, editors, Proceedings of the 4 th Conference on Concept Lattices and Applications (CLA 07), pages 199-212, Montpellier.

[35] Valverde-Albacete, F. J. and Peláez-Moreno, C. (2007b). Galois connections between semimodules and applications in data mining. In S. Kusnetzov and S. Schmidt, editors, Formal Concept Analysis. Proceedings of the 5th International Conference on Formal Concept Analysis, ICFCA 200\%, ClermontFerrand, France, volume 4390 of LNAI, pages 181-196. Springer.

[36] Wille, R. (1995). The basic theorem of Triadic Concept Analysis. Order, $12(2), 149-158$.

[37] Wille, R. (2004). Dyadic Mathematics - Abstractions from Logical Thought, pages 453-498. In 9$]$. 


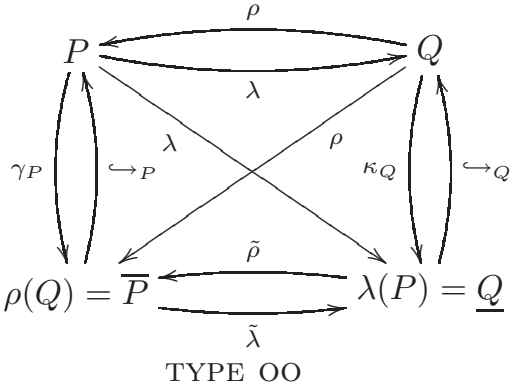

Left Adjunction, $(\lambda, \rho): \mathcal{P} \leftrightharpoons \mathcal{Q}$

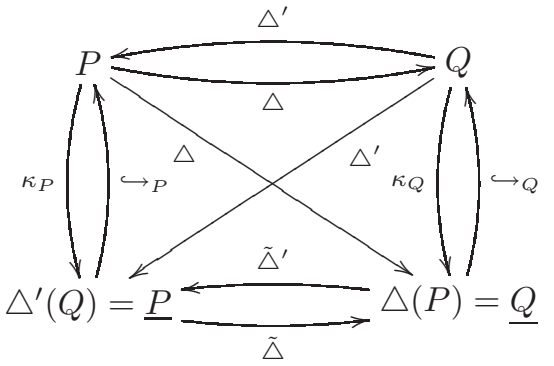

TYPE IO

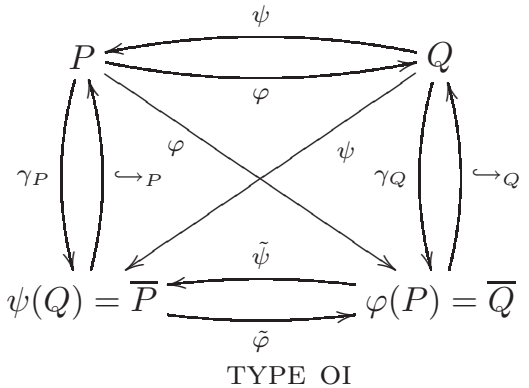

Galois connection: $(\varphi, \psi): \mathcal{P} \triangleleft \mathcal{Q}$

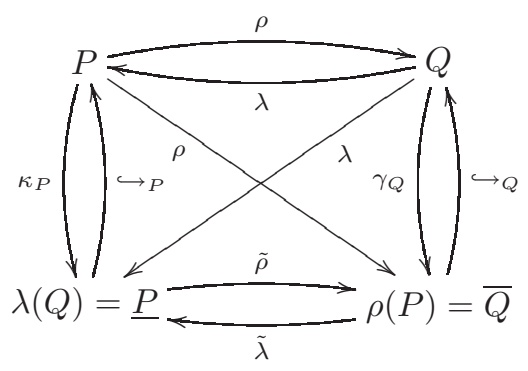

TYPE II

Co-Galois connection, $\left(\triangle, \triangle^{\prime}\right): \mathcal{P} \neg \mathcal{Q} \quad$ Right Adjunction, $(\rho, \lambda): \mathcal{P} \rightleftharpoons \mathcal{Q}$

Figure 1: Diagrams visually depicting the maps and structures involved in the adjunction on the left $(\lambda, \rho): P \leftrightharpoons Q$ (top left), Galois connection $(\varphi, \psi): P \leftrightharpoons Q$ (top right), the co-Galois connection $\left(\triangle, \triangle^{\prime}\right): P \neg Q$ (bottom left) and the adjunction on the right $(\rho, \lambda): P \rightleftharpoons Q$ (bottom right) between two partially ordered sets (adapted from [12, 32]). Closure operators are denoted by $\gamma_{P}, \gamma_{Q}$, interior (kernel) operators by $\kappa_{P}, \kappa_{Q}$, closure systems by $\bar{P}, \bar{Q}$ and interior (kernel) systems by $\underline{P}, \underline{Q}$. 


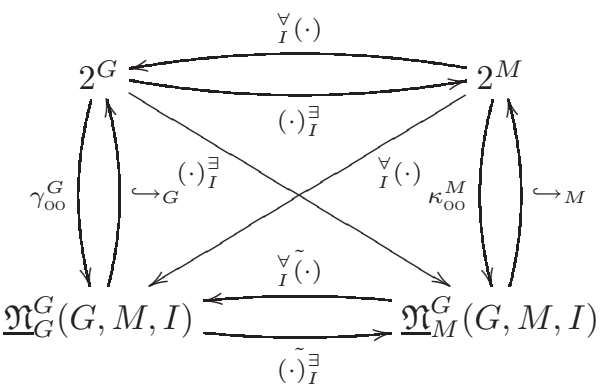

Left Adjunction : $\left[(\cdot)_{I}^{\exists},{ }_{I}^{\forall}(\cdot)\right]: 2^{G} \leftrightharpoons 2^{M}$

$\underline{\mathfrak{B}}_{\mathrm{OO}}(G, M, I) \cong \underline{\mathfrak{N}}^{G}(G, M, I)$

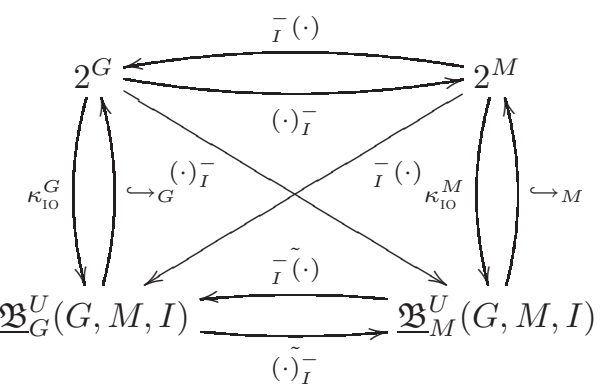

co-Galois Conn.: $\left[(\cdot)_{I}^{-}, \bar{I}(\cdot)\right]: 2^{G} \nabla 2^{M}$

$\underline{\mathfrak{B}}_{\mathrm{IO}}(G, M, I) \equiv \underline{\mathfrak{B}}^{U}(G, M, I)$

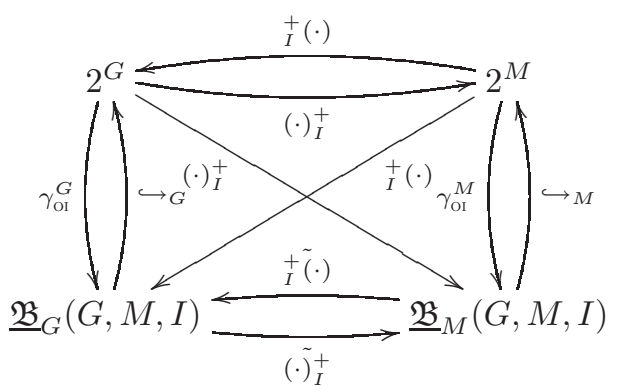

Galois Conn. : $\left[(\cdot)_{I}^{+},{ }_{I}^{+}(\cdot)\right]: 2^{G} \sqcup 2^{M}$

$\underline{\mathfrak{B}}_{\mathrm{OI}}(G, M, I) \cong \underline{\mathfrak{B}}(G, M, I)$

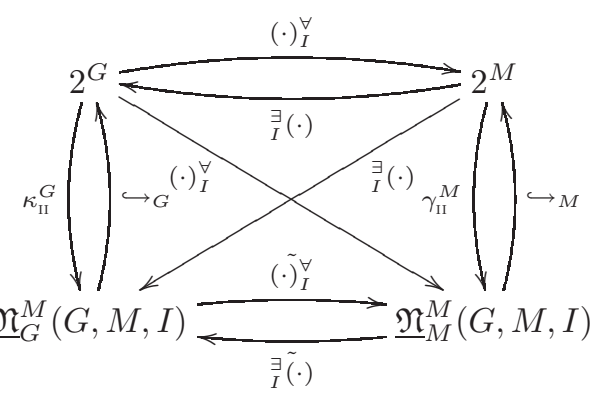

Right adjunction : $\left[(\cdot)_{I}^{\forall},{ }_{I}^{\exists}(\cdot)\right]: 2^{G} \rightleftharpoons 2^{M}$

$\underline{\mathfrak{B}}_{\mathrm{II}}(G, M, I) \cong \underline{\mathfrak{N}}^{M}(G, M, I)$

Figure 2: Summary of notation for describing the broad-sense concept lattices of all Galois connections between posets $2^{G}$ and $2^{M}$ induced by a binary incidence. The notation for closures, kernels and canonical injections is simplified. When FCA supplies a name for a particular set, we use it in the diagram and likewise for the neighbourhood of objects $\underline{\mathfrak{N}}^{G}(G, M, I)$, the neighbourhood of attributes $\underline{\mathfrak{N}}^{M}(G, M, I)$, and the lattice of unrelatedness $\underline{\mathfrak{B}}^{U}(G, M, I)$. Note that each concept lattice splits into a lattice of object sets and a lattice of attribute sets.

$$
C M=\left[\begin{array}{ccc}
5 & 3 & 0 \\
2 & 3 & 1 \\
0 & 2 & 11
\end{array}\right] I^{C M}=\left[\begin{array}{lll}
1 & 1 & 0 \\
0 & 1 & 0 \\
0 & 0 & 1
\end{array}\right]
$$

Figure 3: The count confusion matrix $C M$ and a binarisation of it $I^{C M}$. 


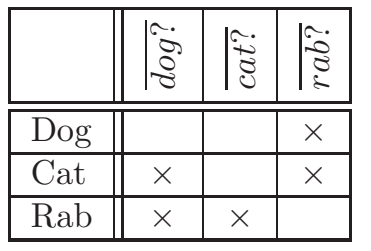

(a) TYPE OO: $\left(G, M^{-}, \Psi\right)$

\begin{tabular}{|c|c|c|c|}
\hline & $\mid \overrightarrow{8}$ & $\mid \tilde{z}$ & $\mid$ \\
\hline$\overline{D o g}$ & $x$ & $x$ & \\
\hline$\overline{\overline{C a t}}$ & & $\times$ & \\
\hline$\overline{R a b}$ & & & $x$ \\
\hline
\end{tabular}

(c) TYPE IO: $\left(G^{-}, M^{-}, I\right)$

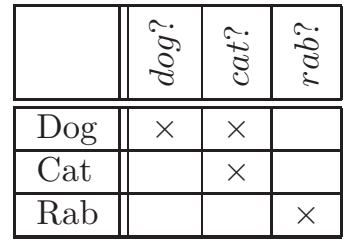

(b) TYPE OI: $(G, M, I)$

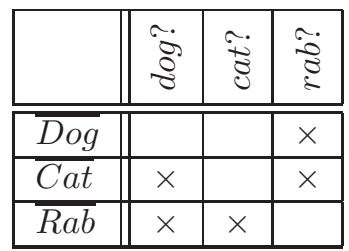

(d) TYPE II: $\left(G^{-}, M, \digamma\right)$

Figure 4: The four broad-sense contexts derived from relation $I^{C M}$. Notation explained in the text. 


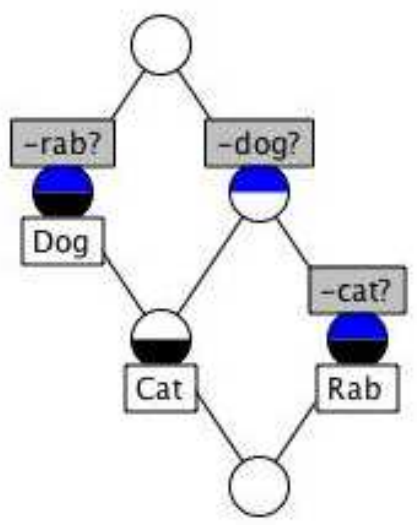

(a) $\underline{\mathfrak{B}}_{\mathrm{OO}}(G, M, I) \cong \underline{\mathfrak{B}}\left(G, M^{-}, \mp\right)$

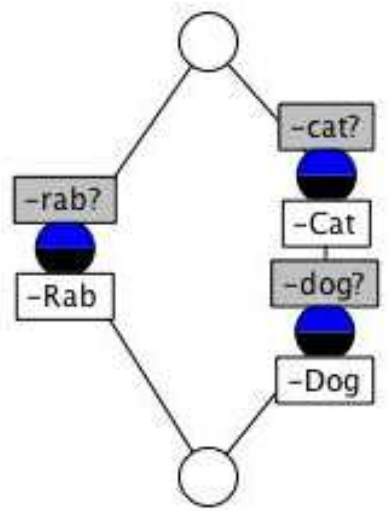

(c) $\underline{\mathfrak{B}}_{\mathrm{IO}}(G, M, I) \cong \underline{\mathfrak{B}}\left(G^{-}, M^{-}, I\right)$

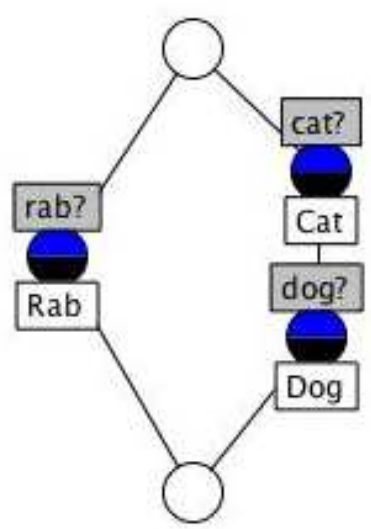

(b) $\underline{\mathfrak{B}}_{\mathrm{OI}}(G, M, I) \cong \underline{\mathfrak{B}}(G, M, I)$

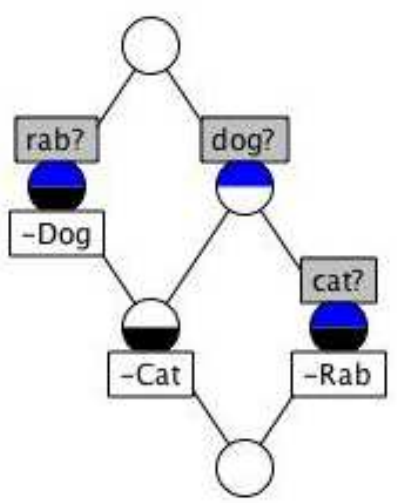

(d) $\underline{\mathfrak{B}}_{\mathrm{II}}(G, M, I) \cong \underline{\mathfrak{B}}\left(G^{-}, M, \Psi\right)$

Figure 5: The four broad-sense lattices derived from relation $I$. Notation explained in the text. 


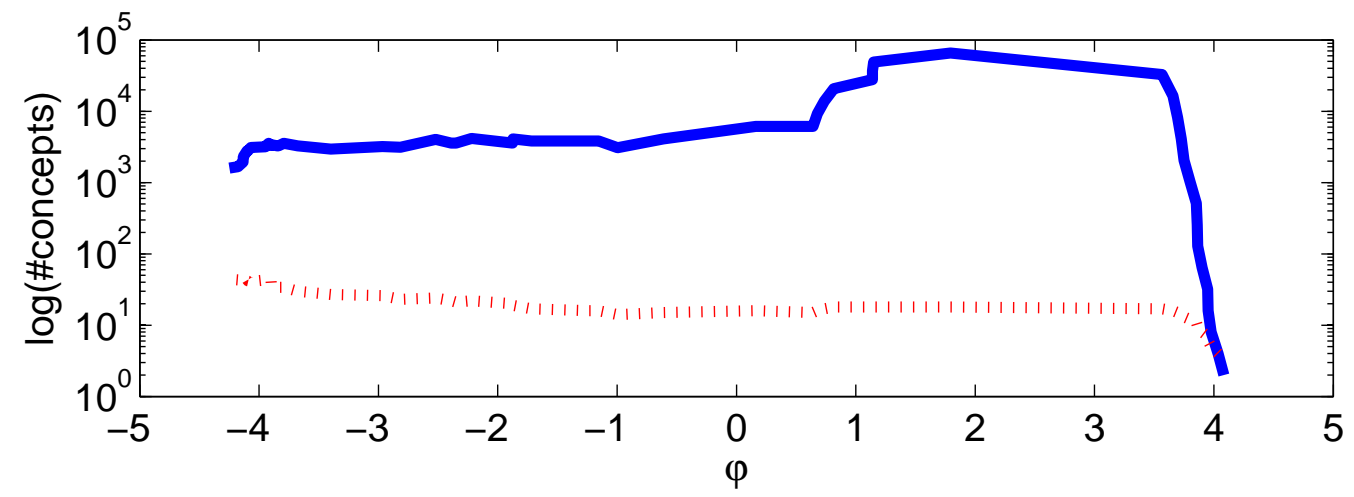

Signal-to-Noise Ratio 12dB.

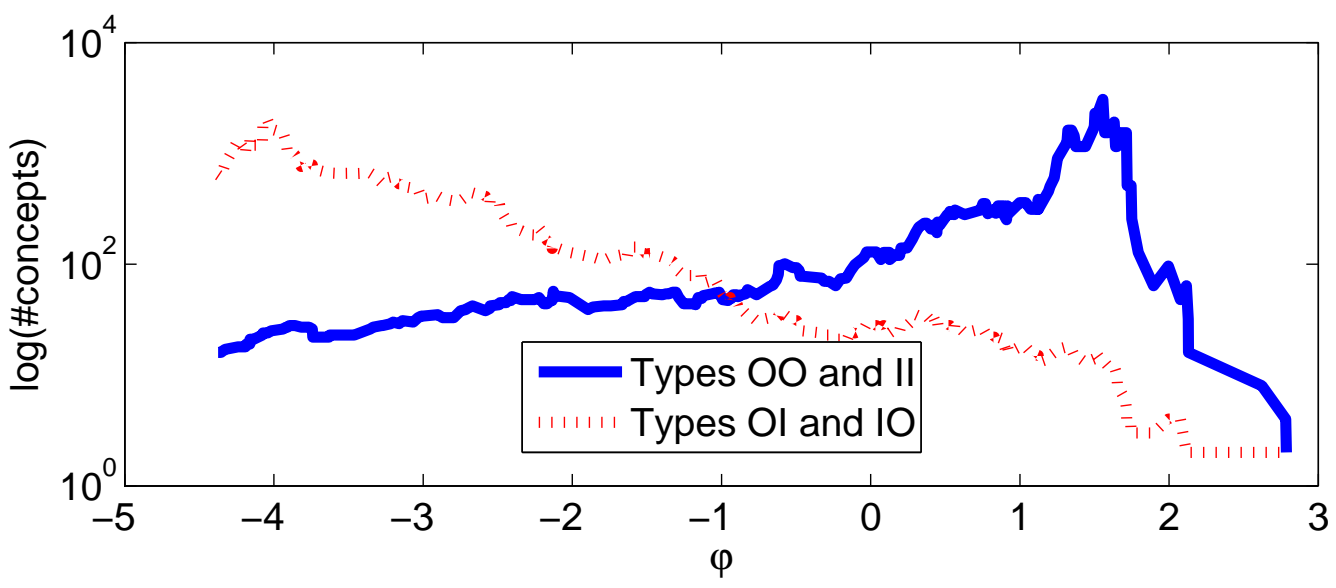

Signal-to-Noise Ratio -12dB.

Figure 6: Number of concepts versus $\varphi$ of the structural lattices of consonant perception confusion matrix on a noisy environment extracted from [25]: Signalto-Noise Ratio $12 \mathrm{~dB}$ (top), Signal-to-Noise Ratio -12dB (bottom). 


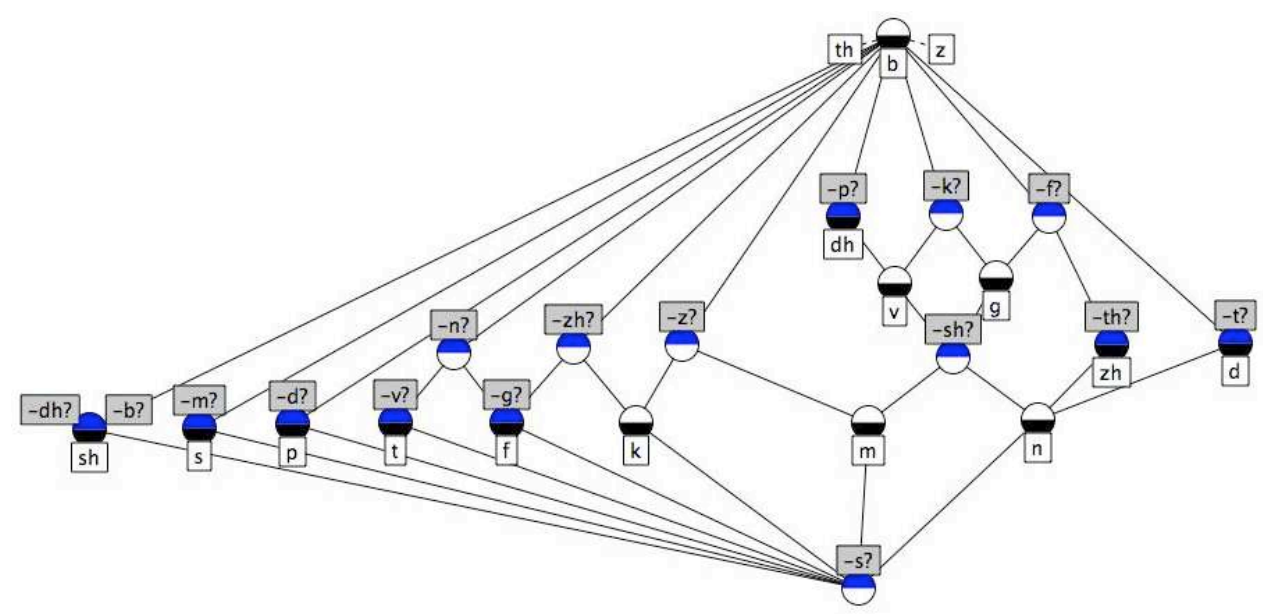

TYPE OO, Left Adjunction: $\underline{\mathfrak{N}}^{G}(G, M, I)$

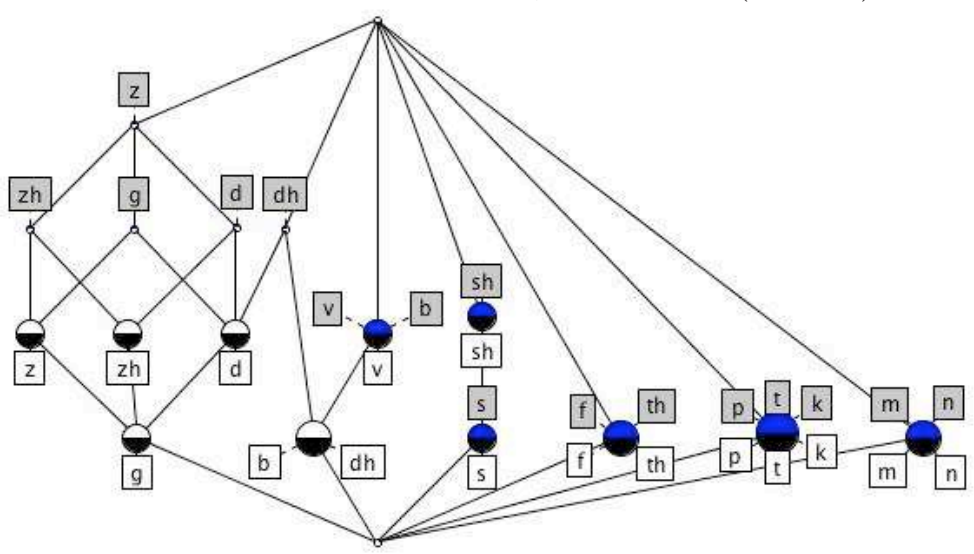

TYPE OI, Galois connection, $\underline{\mathfrak{B}}(G, M, I)$

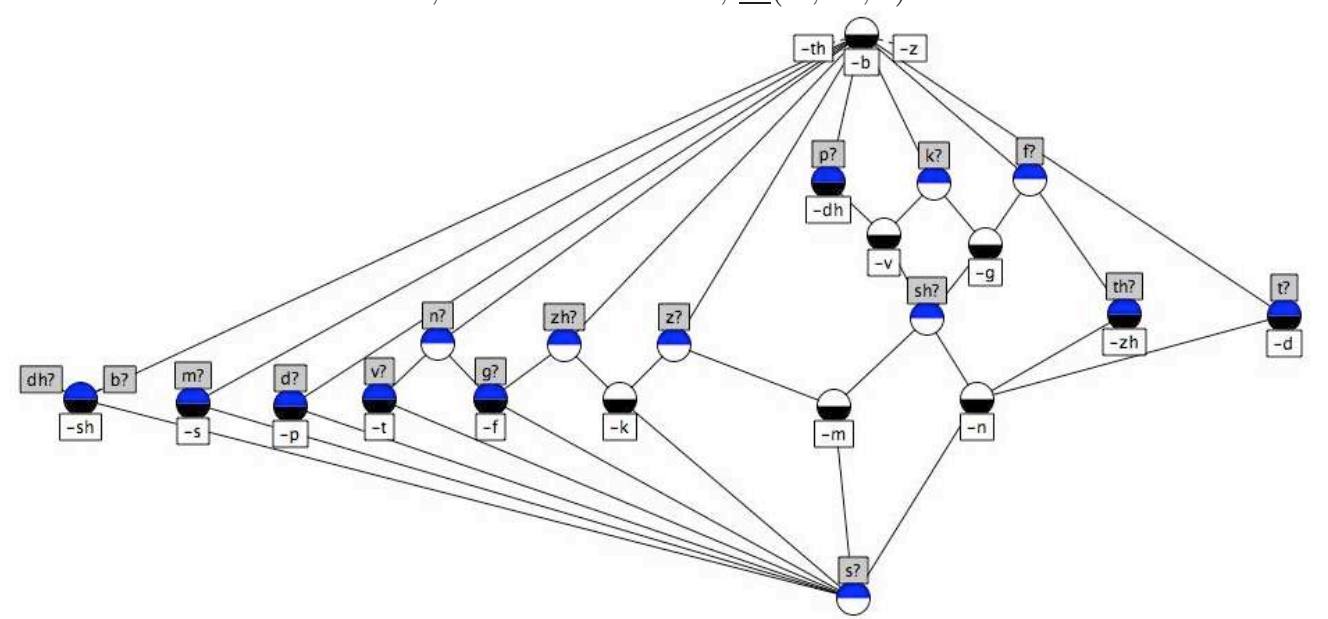

TYPE II, right adjunction, $\underline{\mathfrak{N}}^{M}(G, M, I)$

Figure 7: Broad-sense structural lattic 39 of consonant perception confusion matrix on a noisy environment (Signal-to-Noise Ratio -12dB) extracted from 25]: TYPE OO with $\varphi=-4.16$ (top), TYPE OI with $\varphi=0.99$ (center), TYPE II with $\varphi=-4.16$ (bottom). 